Supporting information for:

\title{
Vapor-Liquid-Equilibria of mixtures containing ethanol, oxygen and nitrogen at elevated pressure and temperature - measured with in situ Raman spectroscopy in microcapillaries
}

Michael H. H. Fechter ${ }^{\mathrm{a}}$, Andreas S. Braeuer ${ }^{\mathrm{a}, \mathrm{b}, *}$

a Institute of Thermal-, Environmental-, and Resources' Process Engineering (ITUN), Technische Universität Bergakademie Freiberg (TUBAF), Leipzigerstr. 28, 09599, Germany

${ }^{\mathrm{b}}$ Zentrum für effiziente Hochtemperatur-Stoffwandlung (ZeHS), Technische Universität Bergakademie Freiberg (TUBAF), am St.-Niclas-Schacht 13, 09599, Germany

*E-mail: andreas.braeuer@tu-freiberg.de

List of content:

I $\quad-\quad$ Calibration results

II - $\quad$ Fitting of binary interaction parameters

III - $\quad$ Modelling of VLE data with Peng-Robinson-EOS

IV - $\quad$ Pseudo-binary Tx-results

V - - Henry's law coefficients

VI - Average percentage errors

VII - VLE data

VIII - List of symbols

IX - $\quad$ References 


\section{I - Calibration results}

The calibration results are given in detail in Table S1: for given pressure and temperature the set molar ratios of ethanol to nitrogen $\left(R_{\mathrm{Eth}-\mathrm{N}_{2}}\right)$, ethanol to oxygen $\left(R_{\mathrm{Eth}-\mathrm{O}_{2}}\right)$ and oxygen to nitrogen $\left(R_{\mathrm{O}_{2}-\mathrm{N}_{2}}\right)$, as well as the respective measured signal ratios $\left(r_{\mathrm{Eth}}{ }_{2}\right.$, $r_{\mathrm{Eth}-\mathrm{O}_{2}}$ and $\left.r_{\mathrm{O}_{2}-\mathrm{N}_{2}}\right)$, and the respective standard uncertainties of the mean $\left(u\left(r_{\mathrm{Eth}-\mathrm{N}_{2}}\right), u\left(r_{\mathrm{Eth}-\mathrm{O}_{2}}\right)\right.$ and $\left.u\left(r_{\mathrm{O}_{2}-\mathrm{N}_{2}}\right)\right)$, calculated as the standard deviations of the measured signal ratios that were obtained by the 32 single spectra each.

Table S1 - calibration results on which the VLE results are based on; the standard uncertainty in terms of pressure is $0.2 \mathrm{MPa}$ and in terms of temperature is $0.3 \mathrm{~K}$.

\begin{tabular}{|c|c|c|c|c|c|c|c|c|c|c|}
\hline$p / \mathrm{MPa}$ & $T / \mathrm{K}$ & $R_{\text {Eth-N2 }}$ & $R_{\text {Eth-O2 }}$ & $R_{\mathrm{O} 2-\mathrm{N} 2}$ & $r_{\text {Eth-N2 }}$ & $r_{\text {Eth-O2 }}$ & $r_{\mathrm{O} 2-\mathrm{N} 2}$ & $u\left(r_{\text {Eth-N2}}\right)$ & $u\left(r_{\text {Eth-O2 } 2}\right)$ & $u\left(r_{\mathrm{O} 2-\mathrm{N} 2}\right)$ \\
\hline 8 & 522 & 10.96 & 43.82 & 0.25 & 213.980 & 737.181 & 0.290 & 7.196 & 85.470 & 0.0352 \\
\hline 8 & 522 & 4.82 & 19.26 & 0.25 & 88.930 & 301.668 & 0.295 & 3.337 & 15.733 & 0.0156 \\
\hline 8 & 522 & 1.81 & 7.22 & 0.25 & 35.909 & 121.226 & 0.296 & 1.120 & 6.488 & 0.0128 \\
\hline 8 & 522 & 1.20 & 4.82 & 0.25 & 23.862 & 80.768 & 0.295 & 0.482 & 4.569 & 0.0126 \\
\hline 8 & 522 & 0.80 & 3.21 & 0.25 & 15.749 & 53.037 & 0.297 & 0.218 & 1.959 & 0.0122 \\
\hline 8 & 522 & 0.52 & 2.06 & 0.25 & 9.798 & 32.766 & 0.299 & 0.398 & 1.656 & 0.0089 \\
\hline 8 & 522 & 0.30 & 1.20 & 0.25 & 5.728 & 19.030 & 0.301 & 0.138 & 0.626 & 0.0064 \\
\hline 8 & 522 & 0.20 & 0.79 & 0.25 & 4.329 & 14.315 & 0.302 & 0.177 & 0.674 & 0.0087 \\
\hline 3 & 512 & 2.80 & 11.20 & 0.25 & 54.966 & 190.421 & 0.289 & 2.548 & 24.206 & 0.0374 \\
\hline 3 & 512 & 1.80 & 7.20 & 0.25 & 35.930 & 123.667 & 0.291 & 1.290 & 12.801 & 0.0308 \\
\hline 3 & 512 & 1.20 & 4.80 & 0.25 & 22.646 & 75.488 & 0.300 & 0.596 & 6.443 & 0.0267 \\
\hline 3 & 512 & 0.80 & 3.20 & 0.25 & 13.671 & 46.435 & 0.294 & 0.697 & 3.934 & 0.0243 \\
\hline 3 & 512 & 0.51 & 2.06 & 0.25 & 9.653 & 32.507 & 0.297 & 0.334 & 2.054 & 0.0152 \\
\hline 3 & 512 & 0.30 & 1.20 & 0.25 & 5.665 & 19.067 & 0.297 & 0.745 & 2.588 & 0.0171 \\
\hline 3 & 512 & 0.19 & 0.76 & 0.25 & 3.388 & 11.711 & 0.289 & 0.321 & 1.079 & 0.0213 \\
\hline 6 & 502 & 4.93 & 19.72 & 0.25 & 89.219 & 305.898 & 0.292 & 6.291 & 28.945 & 0.0268 \\
\hline 6 & 502 & 1.80 & 7.21 & 0.25 & 36.099 & 122.511 & 0.295 & 1.143 & 9.588 & 0.0216 \\
\hline 6 & 502 & 0.80 & 3.20 & 0.25 & 15.539 & 52.334 & 0.297 & 0.385 & 2.698 & 0.0128 \\
\hline 6 & 502 & 0.30 & 1.19 & 0.25 & 5.923 & 20.034 & 0.296 & 0.730 & 2.598 & 0.0112 \\
\hline 5 & 502 & 1.80 & 7.21 & 0.25 & 35.631 & 124.393 & 0.286 & 0.745 & 6.900 & 0.0156 \\
\hline 5 & 502 & 0.80 & 3.20 & 0.25 & 14.645 & 49.784 & 0.294 & 0.478 & 2.820 & 0.0130 \\
\hline 5 & 502 & 0.51 & 2.06 & 0.25 & 9.606 & 32.724 & 0.294 & 0.309 & 1.280 & 0.0113 \\
\hline 5 & 502 & 0.30 & 1.20 & 0.25 & 5.008 & 16.803 & 0.298 & 0.198 & 0.682 & 0.0076 \\
\hline 5 & 502 & 0.14 & 0.55 & 0.25 & 1.642 & 5.546 & 0.296 & 0.133 & 0.427 & 0.0088 \\
\hline 6.5 & 522 & 10.82 & 43.28 & 0.25 & 219.698 & 785.572 & 0.280 & 11.418 & 118.143 & 0.0434 \\
\hline 6.5 & 522 & 4.81 & 19.24 & 0.25 & 97.324 & 330.690 & 0.294 & 1.979 & 26.687 & 0.0255 \\
\hline 6.5 & 522 & 2.79 & 11.17 & 0.25 & 58.506 & 200.928 & 0.291 & 1.254 & 12.639 & 0.0177 \\
\hline 6.5 & 522 & 1.80 & 7.21 & 0.25 & 36.181 & 123.082 & 0.294 & 0.874 & 6.794 & 0.0174 \\
\hline 6.5 & 522 & 1.20 & 4.81 & 0.25 & 23.974 & 81.581 & 0.294 & 0.643 & 3.636 & 0.0103 \\
\hline 6.5 & 522 & 0.80 & 3.21 & 0.25 & 16.019 & 53.560 & 0.299 & 0.422 & 2.184 & 0.0102 \\
\hline 6.5 & 522 & 0.52 & 2.06 & 0.25 & 10.352 & 34.442 & 0.301 & 0.294 & 1.224 & 0.0096 \\
\hline 6.5 & 522 & 0.30 & 1.20 & 0.25 & 5.993 & 19.999 & 0.300 & 0.131 & 0.728 & 0.0086 \\
\hline 6.5 & 522 & 0.17 & 0.66 & 0.25 & 2.878 & 9.559 & 0.301 & 0.157 & 0.577 & 0.0058 \\
\hline
\end{tabular}




\section{II - Fitting of binary interaction parameters}

The binary interaction parameters of the Peng-Robinson-EOS for ethanol/oxygen $\left(K_{\mathrm{Eth}-\mathrm{O}_{2}}\right)$ and ethanol/nitrogen $\left(K_{\mathrm{Eth}-\mathrm{N}_{2}}\right)$ were fitted by a least square approach (Levenberg-Marquardt algorithm) in the temperature range of $T=\{303$ to 463$\} \mathrm{K}$ in steps of $\Delta T=$ $10 \mathrm{~K}$, minimizing the error $\left(F_{\mathrm{T}=\mathrm{c}} \quad.\right)$ of the objective function $(\mathrm{S} 1)$ for each temperature. This function describes the deviation between the experimental pressure $\left(p_{\text {exp }}\right)$ of the isothermal $p x$-data points, given in supporting information $\mathrm{B}$, and the respective calculated pressure obtained by the $p x$-cycle of the PR-EOS, as shown in Figure S2. The results are given in Figure S1 and were fitted by straight lines (coefficients given in main manuscript).

$$
F_{\mathrm{T}=\text { const. }}=\sum_{k=1}^{n p}\left(\frac{p_{\text {exp }, \mathrm{k}}-p_{\text {calc, } \mathrm{k}}}{p_{\text {exp }, \mathrm{k}}}\right)^{2}
$$

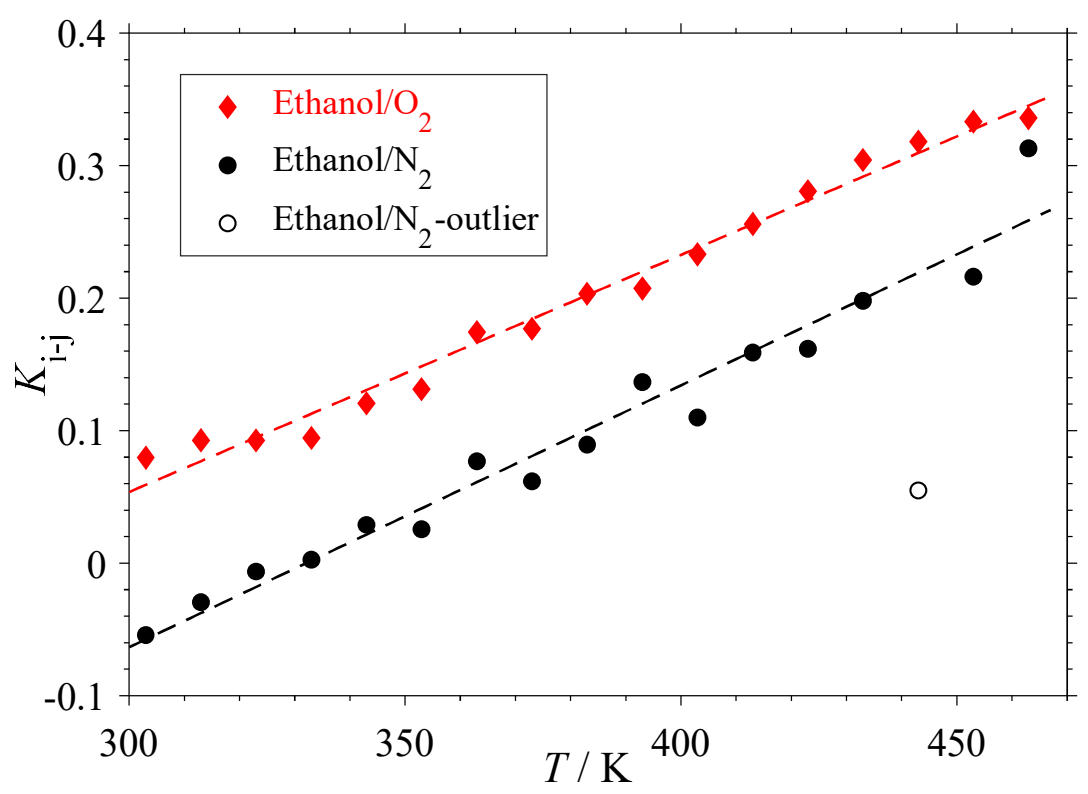

Figure S1 - fitting of binary interaction parameters $\left(K_{\mathrm{i}-\mathrm{j}}\right)$ of the Peng-Robinson-EOS by least square minimization of objective function (S1); an outlier at $443 \mathrm{~K}$ for ethanol/nitrogen was not considered for the linear fit

\section{III - Modelling of VLE data with Peng-Robinson-EOS}

The flowchart that describes the applied modelling of the Peng-Robinson-EOS is shown in Figure S2. Initial parameters are pressure $(p)$ and vapor phase mole fractions $\left(x_{\dot{\mathrm{i}}}^{\mathrm{V}}\right)$, while fixing the temperature $(T)$ and liquid phase mole fractions $\left(x_{\mathrm{i}}^{\mathrm{L}}\right)$. Input parameters for the $p x$ - and $T x$-cycle are hereby the acentric factors $\left(\omega_{\mathrm{i}}\right)$, binary interaction parameters $\left(K_{\mathrm{i}-\mathrm{j}}\right)$, as well as critical temperatures $\left(T_{\text {crit,i }}\right)$ and pressures $\left(p_{\text {crit, } \mathrm{i}}\right)$, to calculate phase-independent pure parameters $\left(\alpha_{\mathrm{i}}, a_{\mathrm{i}-\mathrm{i}}, b_{\mathrm{i}-\mathrm{i}}\right)$ and mixture parameters $\left(a_{\mathrm{i}-\mathrm{j}}\right)$, as well as phasedependent mixture parameters $(a, b)$, fugacity coefficients $(\varphi)$ and molar volumes $(v)$. In the ternary calculation cycle the liquid phase mole fraction of ethanol $\left(x_{\mathrm{Eth}}^{\mathrm{L}}\right)$ is iterated for a given liquid phase oxygen/nitrogen molar ratio $\left(R_{\mathrm{O}_{2}-\mathrm{N}_{2}}^{\mathrm{L}}\right)$, in order to find the matching ternary (isothermal and isobaric) compositions for given temperature and pressure $\left(p_{\text {ter }}\right)$. $\varepsilon$ are termination criteria to either check isofugacity or the deviations between calculated and goal pressures in the $T x$ - and ternary cycles. 


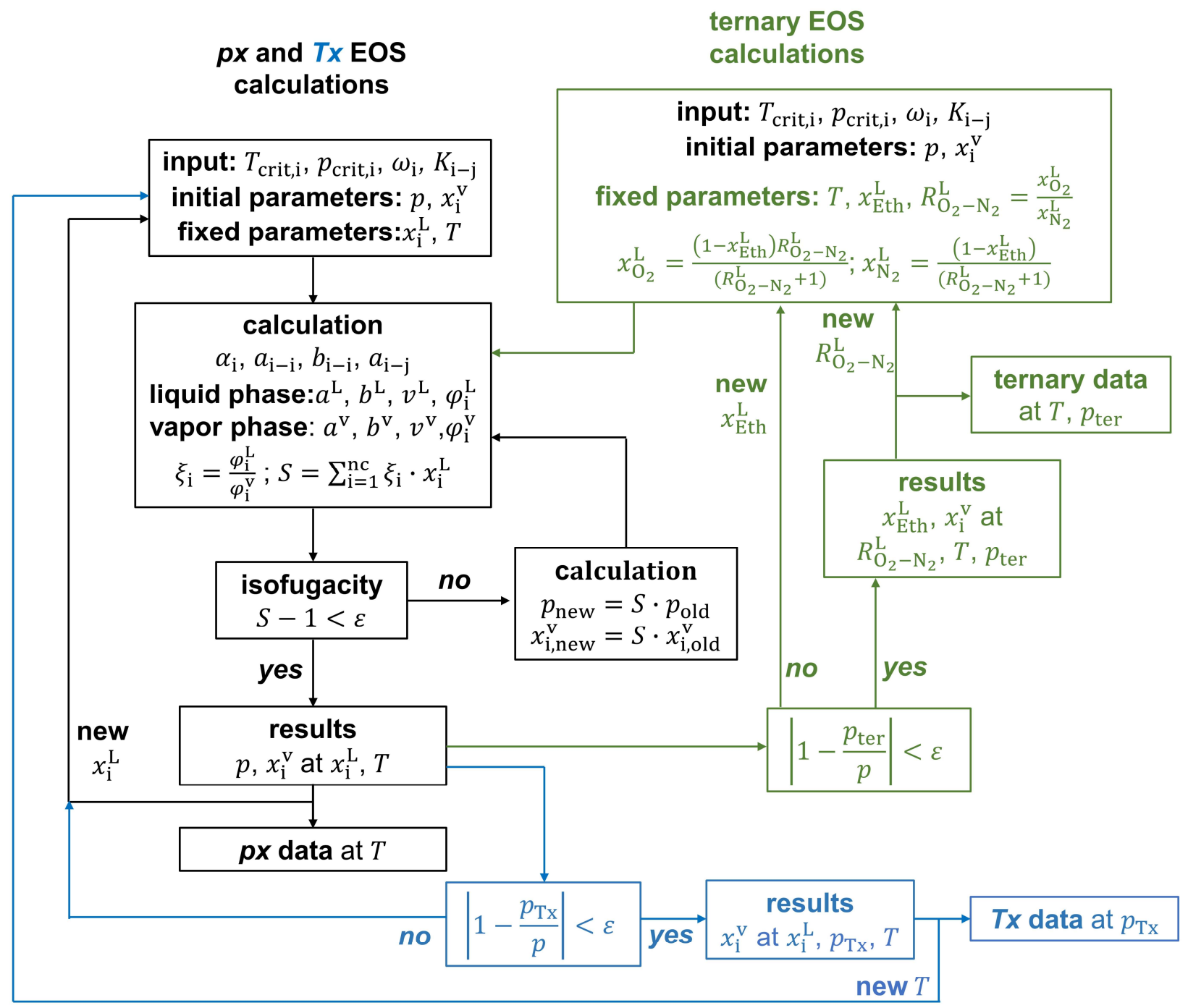

Figure S2: flow chart of the applied modelling approach using the Peng-Robinson EOS 


\section{IV - Pseudo-binary Tx-results}

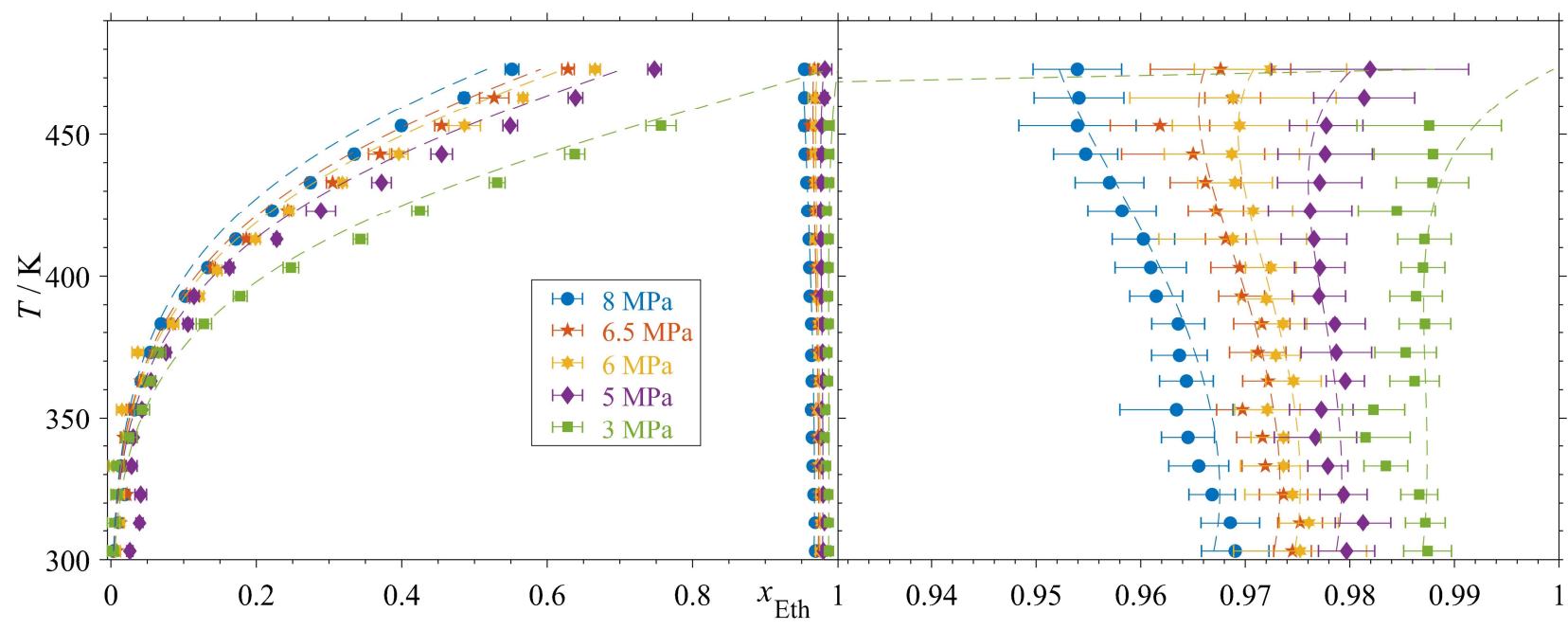

Figure S3 - left chart: pseudo-binary Tx-diagrams at different pressures of ethanol/ gas mix 1; right chart: zoom of liquid phase compositions (bubble lines); (errorbars: standard-deviation of 32 single spectra); (- - Peng-Robinson EOS)

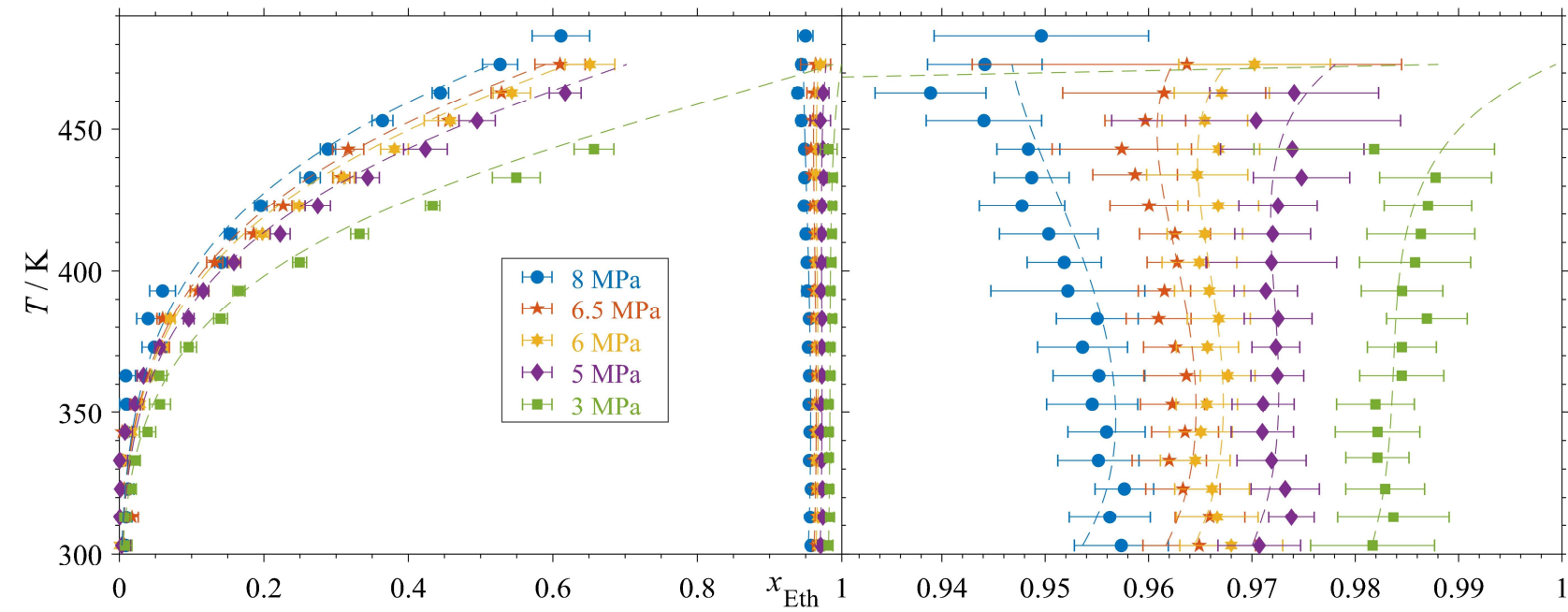

Figure S4 - left chart: pseudo-binary Tx-diagrams at different pressures of ethanol/gas mix 2; right chart: zoom of liquid phase compositions (bubble lines); (errorbars: standard-deviation of 32 single spectra); (- - Peng-Robinson EOS)

\section{V - Henry's law coefficients}

The Henry's law coefficients obtained in this study by the Peng-Robinson-EOS $\left(\mathrm{H}_{\mathrm{O}_{2}}\right)$ are given in Table S2. The results are compared by the absolute deviations $\left(\Delta H_{\mathrm{O}_{2}}\right.$; equation (S2)) to the literature data of Schnabel et al. ${ }^{1}\left({ }^{<}:\right.$adjusted mode, ${ }^{\#}$ : predictive mode) and Malviya et al. $^{2}\left({ }^{+}\right)$.

$$
\Delta H_{\mathrm{O}_{2}}=H_{\mathrm{O}_{2}}-H_{\mathrm{O}_{2}}^{\text {lit }}
$$


Table S2 - derived Henry coefficients $\left(H_{\mathrm{O}_{2}}\right)$ for solubility of oxygen in ethanol and comparison to available literature data by absolute deviations $\left(\Delta H_{\mathrm{O}_{2}}\right)$

\begin{tabular}{ccccc|ccccc}
$T / \mathrm{K}$ & $H_{\mathrm{O}_{2}} / \mathrm{MPa}$ & $\Delta H_{\mathrm{O}_{2}}^{<} / \mathrm{MPa}$ & $\Delta H_{\mathrm{O}_{2}}^{\#} / \mathrm{MPa}$ & $\Delta H_{\mathrm{O}_{2}}^{+} / \mathrm{MPa}$ & $T / \mathrm{K}$ & $H_{\mathrm{O}_{2}} / \mathrm{MPa}$ & $\Delta H_{\mathrm{O}_{2}}^{*} / \mathrm{MPa}$ & $\Delta H_{\mathrm{O}_{2}}^{\#} / \mathrm{MPa}$ & $\Delta H_{\mathrm{O}_{2}}^{+} / \mathrm{MPa}$ \\
\hline 303 & 145.4 & - & - & - & 393 & 148.5 & - & - & -1.4 \\
313 & 151.3 & - & - & -22.2 & 403 & 142.9 & - & - & - \\
323 & 155.6 & -20.7 & -3.7 & - & 413 & 136.5 & - & - & -1.2 \\
333 & 158.6 & - & - & -13.2 & 423 & 129.3 & -1.3 & 5.6 & - \\
343 & 160.1 & - & - & - & 433 & 121.3 & - & - & -2.2 \\
353 & 160.2 & - & - & -9.0 & 443 & 112.8 & - & - & - \\
363 & 159.1 & - & - & - & 453 & 103.7 & - & - & -5.8 \\
373 & 156.6 & -3.5 & 7.5 & -3.4 & 463 & 94.1 & - & - & - \\
383 & 153.1 & - & - & - & 473 & 84.0 & - & - & -9.8 \\
\hline
\end{tabular}

\section{VI - Average percentage errors}

The average percentage errors of pressure $\left(\Delta p_{\mathrm{T}=\text { const. }}\right)$ and vapor phase composition $\left(\Delta x_{\mathrm{Eth}, \mathrm{T}=\mathrm{const}}^{\mathrm{V}}\right)$ are calculated with the following equations (S3) and (S4) for each temperature for the number of available experimental points $(n p)$. The corresponding calculated values are obtained by the px-cycle of the Peng-Robinson-EOS (compare Figure S2). The results are given in Table S3 for the system ethanol/oxygen.

$$
\begin{gathered}
\Delta p_{\mathrm{T}=\text { const. }}=\frac{1}{n p} \cdot \sum_{k=1}^{n p} 100 \% \cdot\left|\left(\frac{p_{\text {exp }, \mathrm{k}}-p_{\text {calc, } \mathrm{k}}}{p_{\text {exp, } \mathrm{k}}}\right)\right| \\
\Delta x_{\mathrm{Eth}, \mathrm{T}=\text { const }}^{\mathrm{V}}=\frac{1}{n p} \cdot \sum_{k=1}^{n p} 100 \% \cdot\left|\left(\frac{x_{\text {exp,Eth, } \mathrm{k}}^{\mathrm{v}}-x_{\text {calc,Eth, } \mathrm{k}}^{\mathrm{v}}}{x_{\text {exp }, \mathrm{Eth}, \mathrm{k}}^{\mathrm{V}}}\right)\right|
\end{gathered}
$$

Table S3 - average percentage errors of pressure and vapor phase composition between model (Peng-Robinson-EOS) and experiments for the system ethanol/oxygen

\begin{tabular}{ccc|ccc}
$T / \mathrm{K}$ & $\Delta p / \%$ & $\Delta x_{\mathrm{Eth}}^{\mathrm{v}} / \%$ & $T / \mathrm{K}$ & $\Delta p / \%$ & $\Delta x_{\mathrm{Eth}}^{\mathrm{v}} / \%$ \\
\hline 303 & 10.2 & 156.8 & 393 & 4.8 & 73.2 \\
313 & 7.9 & 280.7 & 403 & 5.0 & 74.1 \\
323 & 3.3 & 202.6 & 413 & 5.3 & 66.1 \\
333 & 4.7 & 4285.7 & 423 & 5.1 & 62.5 \\
343 & 4.7 & 209.1 & 433 & 4.1 & 60.1 \\
353 & 4.4 & 2547.3 & 443 & 4.2 & 51.9 \\
363 & 7.2 & 563.9 & 453 & 2.7 & 37.8 \\
373 & 2.9 & 87.4 & 463 & 2.3 & 41.2 \\
383 & 4.8 & 87.4 & 473 & 1.9 & 41.3 \\
\hline
\end{tabular}


The VLE data (ordered isothermally) are given in the Tables S4, S5 and S6. The necessary data of Klima (ethanol/nitrogen) that has been used for the Peng-Robinson-EOS and the illustration of ternary VLE data are given in Table S7. The standard uncertainty of the mean mole fraction $u(x)$ is calculated as the standard deviation of 32 mole fractions obtained by 32 single Raman spectra. As stated in the calibration section, the standard uncertainty in terms of pressure is $0.2 \mathrm{MPa}$ and in terms of temperature is $0.3 \mathrm{~K}$. All data are furthermore ordered as ternary VLE data in Table S8.

Table S4 - VLE data of the binary system ethanol/oxygen; $u\left(x_{\mathrm{Eth}}^{\mathrm{L}}\right)=u\left(x_{\mathrm{O}_{2}}^{\mathrm{L}}\right)$ and $u\left(x_{\mathrm{Eth}}^{\mathrm{V}}\right)=u\left(x_{\mathrm{O}_{2}}^{\mathrm{v}}\right)$

\begin{tabular}{|c|c|c|c|c|c|c|c|}
\hline$p / \mathrm{MPa}$ & $T / \mathrm{K}$ & $x_{\text {Eth }}^{\mathrm{L}}$ & $x_{0_{2}}^{\mathrm{L}}$ & $x_{\mathrm{Eth}}^{\mathrm{v}}$ & $x_{\mathrm{O}_{2}}^{v}$ & $u\left(x_{\mathrm{Eth}}^{\mathrm{L}}\right)$ & $u\left(x_{\mathrm{Eth}}^{\mathrm{v}}\right)$ \\
\hline 3 & 303 & 0.98305 & 0.01695 & 0.00087 & 0.99913 & 0.00301 & 0.00850 \\
\hline 5 & 303 & 0.97032 & 0.02968 & 0.01872 & 0.98128 & 0.00327 & 0.01035 \\
\hline 6 & 303 & 0.96385 & 0.03615 & 0.01735 & 0.98265 & 0.00311 & 0.00707 \\
\hline 6.5 & 303 & 0.96148 & 0.03852 & 0.00961 & 0.99039 & 0.00314 & 0.01944 \\
\hline 8 & 303 & 0.95371 & 0.04629 & 0.00825 & 0.99175 & 0.00331 & 0.01010 \\
\hline 3 & 313 & 0.98319 & 0.01681 & 0.00165 & 0.99835 & 0.00393 & 0.00863 \\
\hline 5 & 313 & 0.96983 & 0.03017 & 0.01749 & 0.98251 & 0.00389 & 0.01138 \\
\hline 6 & 313 & 0.96550 & 0.03450 & 0.00106 & 0.99894 & 0.00235 & 0.00717 \\
\hline 6.5 & 313 & 0.96212 & 0.03788 & 0.00560 & 0.99440 & 0.00351 & 0.01507 \\
\hline 8 & 313 & 0.95395 & 0.04605 & 0.00512 & 0.99488 & 0.00401 & 0.01103 \\
\hline 3 & 323 & 0.98259 & 0.01741 & 0.00238 & 0.99762 & 0.00403 & 0.01708 \\
\hline 5 & 323 & 0.96829 & 0.03171 & 0.01477 & 0.98523 & 0.00367 & 0.00772 \\
\hline 6 & 323 & 0.96370 & 0.03630 & 0.01126 & 0.98874 & 0.00237 & 0.00800 \\
\hline 6.5 & 323 & 0.96180 & 0.03820 & 0.00576 & 0.99424 & 0.00276 & 0.01758 \\
\hline 8 & 323 & 0.95120 & 0.04880 & 0.00789 & 0.99211 & 0.00399 & 0.01478 \\
\hline 3 & 333 & 0.98056 & 0.01944 & 0.00138 & 0.99862 & 0.00331 & 0.01644 \\
\hline 5 & 333 & 0.96785 & 0.03215 & 0.00008 & 0.99992 & 0.00309 & 0.00982 \\
\hline 6 & 333 & 0.96303 & 0.03697 & 0.01264 & 0.98736 & 0.00250 & 0.00871 \\
\hline 6.5 & 333 & 0.96062 & 0.03938 & 0.00448 & 0.99552 & 0.00317 & 0.01887 \\
\hline 8 & 333 & 0.95059 & 0.04941 & 0.00777 & 0.99223 & 0.00311 & 0.01458 \\
\hline 3 & 343 & 0.98361 & 0.01639 & 0.01643 & 0.98357 & 0.00291 & 0.00928 \\
\hline 5 & 343 & 0.96907 & 0.03093 & 0.02520 & 0.97480 & 0.00287 & 0.01104 \\
\hline 6 & 343 & 0.96357 & 0.03643 & 0.03264 & 0.96736 & 0.00200 & 0.00737 \\
\hline 6.5 & 343 & 0.96109 & 0.03891 & 0.03048 & 0.96952 & 0.00312 & 0.00961 \\
\hline 8 & 343 & 0.95078 & 0.04922 & 0.00272 & 0.99728 & 0.00295 & 0.00893 \\
\hline 3 & 353 & 0.98272 & 0.01728 & 0.02049 & 0.97951 & 0.00295 & 0.01145 \\
\hline 5 & 353 & 0.96961 & 0.03039 & 0.03471 & 0.96529 & 0.00320 & 0.01360 \\
\hline 6 & 353 & 0.96290 & 0.03710 & 0.00186 & 0.99814 & 0.00261 & 0.00917 \\
\hline 6.5 & 353 & 0.96010 & 0.03990 & 0.04521 & 0.95479 & 0.00334 & 0.00696 \\
\hline 8 & 353 & 0.95074 & 0.04926 & 0.00022 & 0.99978 & 0.00285 & 0.01285 \\
\hline 3 & 363 & 0.98717 & 0.01283 & 0.03695 & 0.96305 & 0.00335 & 0.02207 \\
\hline 5 & 363 & 0.97167 & 0.02833 & 0.06825 & 0.93175 & 0.00335 & 0.01219 \\
\hline 6 & 363 & 0.96409 & 0.03591 & 0.06515 & 0.93485 & 0.00266 & 0.00871 \\
\hline 6.5 & 363 & 0.96262 & 0.03738 & 0.05439 & 0.94561 & 0.00353 & 0.01262 \\
\hline 8 & 363 & 0.95222 & 0.04778 & 0.00142 & 0.99858 & 0.00252 & 0.01488 \\
\hline 3 & 373 & 0.98373 & 0.01627 & 0.10973 & 0.89027 & 0.00652 & 0.02708 \\
\hline 5 & 373 & 0.96932 & 0.03068 & 0.08114 & 0.91886 & 0.00391 & 0.01186 \\
\hline 6 & 373 & 0.96467 & 0.03533 & 0.06714 & 0.93286 & 0.00148 & 0.00817 \\
\hline 6.5 & 373 & 0.96219 & 0.03781 & 0.06669 & 0.93331 & 0.00239 & 0.01059 \\
\hline 8 & 373 & 0.95159 & 0.04841 & 0.03257 & 0.96743 & 0.00319 & 0.01294 \\
\hline 3 & 383 & 0.98572 & 0.01428 & 0.15958 & 0.84042 & 0.00325 & 0.00931 \\
\hline 5 & 383 & 0.97086 & 0.02914 & 0.08386 & 0.91614 & 0.00348 & 0.01685 \\
\hline 6 & 383 & 0.96464 & 0.03536 & 0.07009 & 0.92991 & 0.00183 & 0.00906 \\
\hline 6.5 & 383 & 0.96191 & 0.03809 & 0.08807 & 0.91193 & 0.00296 & 0.01046 \\
\hline 8 & 383 & 0.95011 & 0.04989 & 0.06430 & 0.93570 & 0.00207 & 0.00923 \\
\hline 3 & 393 & 0.98524 & 0.01476 & 0.21176 & 0.78824 & 0.00457 & 0.01273 \\
\hline 5 & 393 & 0.96903 & 0.03097 & 0.13429 & 0.86571 & 0.00365 & 0.01483 \\
\hline 6 & 393 & 0.96361 & 0.03639 & 0.09583 & 0.90417 & 0.00210 & 0.00831 \\
\hline 6.5 & 393 & 0.96092 & 0.03908 & 0.13083 & 0.86917 & 0.00264 & 0.01355 \\
\hline 8 & 393 & 0.94805 & 0.05195 & 0.09806 & 0.90194 & 0.00254 & 0.02129 \\
\hline 3 & 403 & 0.98639 & 0.01361 & 0.23464 & 0.76536 & 0.00270 & 0.01421 \\
\hline 5 & 403 & 0.97023 & 0.02977 & 0.17012 & 0.82988 & 0.00356 & 0.01724 \\
\hline 6 & 403 & 0.96453 & 0.03547 & 0.16538 & 0.83462 & 0.00195 & 0.01002 \\
\hline 6.5 & 403 & 0.95839 & 0.04161 & 0.14475 & 0.85525 & 0.00619 & 0.01276 \\
\hline 8 & 403 & 0.94916 & 0.05084 & 0.12122 & 0.87878 & 0.00186 & 0.01296 \\
\hline 3 & 413 & 0.98714 & 0.01286 & 0.34656 & 0.65344 & 0.00642 & 0.01844 \\
\hline 5 & 413 & 0.97133 & 0.02867 & 0.25146 & 0.74854 & 0.00493 & 0.03568 \\
\hline
\end{tabular}




\begin{tabular}{cccccccc}
6 & 413 & 0.96414 & 0.03586 & 0.19910 & 0.80090 & 0.00220 & 0.01055 \\
6.5 & 413 & 0.95805 & 0.04195 & 0.19675 & 0.80325 & 0.00485 & 0.01153 \\
8 & 413 & 0.94872 & 0.05128 & 0.14198 & 0.85802 & 0.00268 & 0.01124 \\
3 & 423 & 0.98920 & 0.01080 & 0.44482 & 0.55518 & 0.00514 & 0.02383 \\
5 & 423 & 0.96977 & 0.03023 & 0.31731 & 0.68269 & 0.00481 & 0.01644 \\
6 & 423 & 0.96356 & 0.03644 & 0.28433 & 0.71567 & 0.00277 & 0.01103 \\
6.5 & 423 & 0.96059 & 0.03941 & 0.24606 & 0.75394 & 0.00420 & 0.00665 \\
8 & 423 & 0.94836 & 0.05164 & 0.15932 & 0.84068 & 0.00234 & 0.00949 \\
3 & 433 & 0.98971 & 0.01029 & 0.57413 & 0.42587 & 0.00566 & 0.02105 \\
5 & 433 & 0.97134 & 0.02866 & 0.37585 & 0.62415 & 0.00349 & 0.01433 \\
6 & 433 & 0.96423 & 0.03577 & 0.35153 & 0.64847 & 0.00295 & 0.01120 \\
6.5 & 433 & 0.95951 & 0.04049 & 0.29160 & 0.70840 & 0.00525 & 0.02682 \\
8 & 433 & 0.94809 & 0.05191 & 0.18230 & 0.81770 & 0.00305 & 0.01901 \\
3 & 443 & 0.99202 & 0.00798 & 0.69988 & 0.30012 & 0.00514 & 0.03816 \\
5 & 443 & 0.97146 & 0.02854 & 0.47582 & 0.52418 & 0.00689 & 0.01909 \\
6 & 443 & 0.96476 & 0.03524 & 0.43103 & 0.56897 & 0.00301 & 0.02256 \\
6.5 & 443 & 0.95879 & 0.04121 & 0.36204 & 0.63796 & 0.00549 & 0.02789 \\
8 & 443 & 0.94664 & 0.05336 & 0.22793 & 0.77207 & 0.00568 & 0.03738 \\
3 & 453 & 0.98998 & 0.01002 & 0.84709 & 0.15291 & 0.01979 & 0.01816 \\
5 & 453 & 0.97458 & 0.02542 & 0.56023 & 0.43977 & 0.00665 & 0.04116 \\
6 & 453 & 0.96651 & 0.03349 & 0.49923 & 0.50077 & 0.00459 & 0.02390 \\
6.5 & 453 & 0.95836 & 0.04164 & 0.45564 & 0.54436 & 0.00446 & 0.01677 \\
8 & 453 & 0.94597 & 0.05403 & 0.33240 & 0.66760 & 0.00630 & 0.02439 \\
5 & 463 & 0.97544 & 0.02456 & 0.65298 & 0.34702 & 0.01050 & 0.02462 \\
6 & 463 & 0.96706 & 0.03294 & 0.60293 & 0.39707 & 0.00529 & 0.01712 \\
6.5 & 463 & 0.95699 & 0.04301 & 0.54422 & 0.45578 & 0.00561 & 0.01233 \\
8 & 463 & 0.94480 & 0.05520 & 0.46537 & 0.53463 & 0.00428 & 0.04557 \\
6 & 473 & 0.96931 & 0.03069 & 0.70215 & 0.29785 & 0.01117 & 0.02542 \\
6.5 & 473 & 0.96259 & 0.03741 & 0.62858 & 0.37142 & 0.00922 & 0.00901 \\
8 & 473 & 0.94320 & 0.05680 & 0.55807 & 0.44193 & 0.00325 & 0.04205 \\
\hline & & & & & & & \\
& & & & & & &
\end{tabular}

Table S5 - VLE data of the system ethanol/gas mix $1 ; u\left(x_{\mathrm{Eth}}^{\mathrm{L}}\right)=u\left(x_{\mathrm{gas}}^{\mathrm{L}}\right)$ and $u\left(x_{\mathrm{Eth}}^{\mathrm{V}}\right)=u\left(x_{\mathrm{gas}}^{\mathrm{V}}\right)$

\begin{tabular}{|c|c|c|c|c|c|c|c|c|c|c|c|c|c|c|c|}
\hline$p / \mathrm{MPa}$ & $T / \mathrm{K}$ & $x_{\text {Eth }}^{\mathrm{L}}$ & $x_{\text {gas }}^{\mathrm{L}}$ & $x_{0_{2}}^{\mathrm{L}}$ & $x_{\mathrm{N}_{2}}^{\mathrm{L}}$ & $x_{\mathrm{Eth}}^{\mathrm{v}}$ & $x_{\mathrm{gas}}^{\mathrm{v}}$ & $x_{0_{2}}^{v}$ & $x_{\mathrm{N}_{2}}^{\mathrm{v}}$ & $u\left(x_{\mathrm{Eth}}^{\mathrm{L}}\right)$ & $u\left(x_{\mathrm{Eth}}^{\mathrm{v}}\right)$ & $u\left(x_{0_{2}}^{\mathrm{L}}\right)$ & $u\left(x_{0_{2}}^{v}\right)$ & $u\left(x_{\mathrm{N}_{2}}^{\mathrm{L}}\right)$ & $u\left(x_{N_{2}}^{v}\right)$ \\
\hline 3 & 303 & 0.98744 & 0.01256 & 0.00236 & 0.01020 & 0.00264 & 0.99736 & 0.19499 & 0.80237 & 0.00229 & 0.00748 & 0.00175 & 0.00230 & 0.01416 & 0.01655 \\
\hline 5 & 303 & 0.97969 & 0.02031 & 0.00487 & 0.01543 & 0.02602 & 0.97398 & 0.18363 & 0.79035 & 0.00269 & 0.00433 & 0.00181 & 0.00188 & 0.00920 & 0.00902 \\
\hline 6 & 303 & 0.97524 & 0.02476 & 0.00569 & 0.01906 & 0.00613 & 0.99387 & 0.18492 & 0.80895 & 0.00636 & 0.00691 & 0.00206 & 0.00548 & 0.01198 & 0.01020 \\
\hline 6.5 & 303 & 0.97452 & 0.02548 & 0.00548 & 0.02000 & 0.00790 & 0.99210 & 0.18711 & 0.80499 & 0.00180 & 0.00227 & 0.00172 & 0.00106 & 0.00410 & 0.00408 \\
\hline 8 & 303 & 0.96904 & 0.03096 & 0.00743 & 0.02353 & 0.00328 & 0.99672 & 0.18980 & 0.80692 & 0.00323 & 0.00323 & 0.00251 & 0.00209 & 0.00841 & 0.00849 \\
\hline 3 & 313 & 0.98722 & 0.01278 & 0.00245 & 0.01033 & 0.00457 & 0.99543 & 0.18342 & 0.81201 & 0.00190 & 0.00938 & 0.00129 & 0.00148 & 0.01685 & 0.01871 \\
\hline 5 & 313 & 0.98127 & 0.01873 & 0.00340 & 0.01534 & 0.03928 & 0.96072 & 0.18522 & 0.77550 & 0.00265 & 0.00496 & 0.00163 & 0.00204 & 0.01304 & 0.01252 \\
\hline 6 & 313 & 0.97609 & 0.02391 & 0.00568 & 0.01823 & 0.01227 & 0.98773 & 0.18123 & 0.80651 & 0.00282 & 0.00620 & 0.00124 & 0.00243 & 0.01324 & 0.01368 \\
\hline 6.5 & 313 & 0.97525 & 0.02475 & 0.00463 & 0.02012 & 0.01233 & 0.98767 & 0.19070 & 0.79697 & 0.00214 & 0.00523 & 0.00109 & 0.00186 & 0.00812 & 0.00934 \\
\hline 8 & 313 & 0.96857 & 0.03143 & 0.00713 & 0.02431 & 0.01042 & 0.98958 & 0.18807 & 0.80151 & 0.00280 & 0.00515 & 0.00221 & 0.00141 & 0.00976 & 0.00834 \\
\hline 3 & 323 & 0.98664 & 0.01336 & 0.00300 & 0.01036 & 0.00767 & 0.99233 & 0.18512 & 0.80720 & 0.00176 & 0.00765 & 0.00176 & 0.00146 & 0.01462 & 0.01705 \\
\hline 5 & 323 & 0.97940 & 0.02060 & 0.00499 & 0.01561 & 0.04098 & 0.95902 & 0.18214 & 0.77688 & 0.00225 & 0.00796 & 0.00139 & 0.00165 & 0.01188 & 0.01306 \\
\hline 6 & 323 & 0.97452 & 0.02548 & 0.00640 & 0.01908 & 0.01097 & 0.98903 & 0.18218 & 0.80684 & 0.00456 & 0.00770 & 0.00162 & 0.00367 & 0.01034 & 0.01240 \\
\hline 6.5 & 323 & 0.97367 & 0.02633 & 0.00524 & 0.02109 & 0.02199 & 0.97801 & 0.18697 & 0.79104 & 0.00231 & 0.00376 & 0.00124 & 0.00183 & 0.00714 & 0.00861 \\
\hline 8 & 323 & 0.96682 & 0.03318 & 0.00811 & 0.02506 & 0.01868 & 0.98132 & 0.18515 & 0.79617 & 0.00222 & 0.00446 & 0.00188 & 0.00140 & 0.00895 & 0.00924 \\
\hline 3 & 333 & 0.98345 & 0.01655 & 0.00377 & 0.01278 & 0.00742 & 0.99258 & 0.18443 & 0.80815 & 0.00210 & 0.01045 & 0.00174 & 0.00145 & 0.01439 & 0.01743 \\
\hline 5 & 333 & 0.97790 & 0.02210 & 0.00679 & 0.01530 & 0.02845 & 0.97155 & 0.18930 & 0.78225 & 0.00191 & 0.00752 & 0.00170 & 0.00134 & 0.01203 & 0.01293 \\
\hline 6 & 333 & 0.97365 & 0.02635 & 0.00718 & 0.01918 & 0.00369 & 0.99631 & 0.18454 & 0.81177 & 0.00409 & 0.00769 & 0.00170 & 0.00357 & 0.01102 & 0.01400 \\
\hline 6.5 & 333 & 0.97192 & 0.02808 & 0.00658 & 0.02150 & 0.01870 & 0.98130 & 0.18438 & 0.79691 & 0.00223 & 0.00248 & 0.00145 & 0.00168 & 0.00513 & 0.00551 \\
\hline 8 & 333 & 0.96555 & 0.03445 & 0.00935 & 0.02510 & 0.01337 & 0.98663 & 0.18249 & 0.80414 & 0.00287 & 0.00438 & 0.00207 & 0.00191 & 0.01104 & 0.01191 \\
\hline 3 & 343 & 0.98150 & 0.01850 & 0.00484 & 0.01366 & 0.02512 & 0.97488 & 0.17898 & 0.79590 & 0.00427 & 0.01070 & 0.00191 & 0.00358 & 0.01889 & 0.02093 \\
\hline 5 & 343 & 0.97672 & 0.02328 & 0.00596 & 0.01733 & 0.03063 & 0.96937 & 0.18409 & 0.78528 & 0.00394 & 0.00544 & 0.00237 & 0.00229 & 0.01130 & 0.00932 \\
\hline 6 & 343 & 0.97365 & 0.02635 & 0.00675 & 0.01960 & 0.02408 & 0.97592 & 0.18352 & 0.79239 & 0.00310 & 0.00705 & 0.00153 & 0.00238 & 0.01416 & 0.01351 \\
\hline 6.5 & 343 & 0.97165 & 0.02835 & 0.00724 & 0.02111 & 0.01761 & 0.98239 & 0.18788 & 0.79451 & 0.00247 & 0.00455 & 0.00192 & 0.00162 & 0.00906 & 0.01022 \\
\hline 8 & 343 & 0.96452 & 0.03548 & 0.00910 & 0.02638 & 0.02348 & 0.97652 & 0.18787 & 0.78866 & 0.00254 & 0.00533 & 0.00174 & 0.00173 & 0.01048 & 0.01063 \\
\hline 3 & 353 & 0.98226 & 0.01774 & 0.00514 & 0.01260 & 0.04272 & 0.95728 & 0.18282 & 0.77446 & 0.00299 & 0.01026 & 0.00207 & 0.00237 & 0.02284 & 0.02546 \\
\hline 5 & 353 & 0.97727 & 0.02273 & 0.00570 & 0.01703 & 0.04257 & 0.95743 & 0.17976 & 0.77766 & 0.00304 & 0.00461 & 0.00229 & 0.00173 & 0.00736 & 0.00934 \\
\hline 6 & 353 & 0.97211 & 0.02789 & 0.00770 & 0.02019 & 0.01516 & 0.98484 & 0.18203 & 0.80281 & 0.00313 & 0.00741 & 0.00174 & 0.00215 & 0.01179 & 0.01223 \\
\hline 6.5 & 353 & 0.96973 & 0.03027 & 0.00791 & 0.02236 & 0.02494 & 0.97506 & 0.18508 & 0.78998 & 0.00247 & 0.00382 & 0.00180 & 0.00166 & 0.00698 & 0.00763 \\
\hline 8 & 353 & 0.96341 & 0.03659 & 0.00970 & 0.02689 & 0.03229 & 0.96771 & 0.18575 & 0.78196 & 0.00541 & 0.00687 & 0.00325 & 0.00324 & 0.00856 & 0.00924 \\
\hline 3 & 363 & 0.98619 & 0.01381 & 0.00286 & 0.01095 & 0.05254 & 0.94746 & 0.18787 & 0.75958 & 0.00236 & 0.00844 & 0.00168 & 0.00165 & 0.01698 & 0.01612 \\
\hline 5 & 363 & 0.97957 & 0.02043 & 0.00333 & 0.01710 & 0.05503 & 0.94497 & 0.17918 & 0.76579 & 0.00183 & 0.00643 & 0.00168 & 0.00157 & 0.00979 & 0.00963 \\
\hline 6 & 363 & 0.97462 & 0.02538 & 0.00454 & 0.02085 & 0.04663 & 0.95337 & 0.17749 & 0.77588 & 0.00265 & 0.00750 & 0.00159 & 0.00170 & 0.01268 & 0.01232 \\
\hline 6.5 & 363 & 0.97218 & 0.02782 & 0.00408 & 0.02374 & 0.04107 & 0.95893 & 0.18328 & 0.77565 & 0.00243 & 0.00334 & 0.00206 & 0.00179 & 0.00711 & 0.00817 \\
\hline 8 & 363 & 0.96437 & 0.03563 & 0.00751 & 0.02812 & 0.04159 & 0.95841 & 0.18434 & 0.77407 & 0.00257 & 0.00351 & 0.00228 & 0.00159 & 0.00738 & 0.00746 \\
\hline 3 & 373 & 0.98534 & 0.01466 & 0.00187 & 0.01279 & 0.06601 & 0.93399 & 0.18089 & 0.75309 & 0.00294 & 0.00932 & 0.00188 & 0.00220 & 0.01599 & 0.01759 \\
\hline 5 & 373 & 0.97872 & 0.02128 & 0.00384 & 0.01745 & 0.07575 & 0.92425 & 0.17444 & 0.74981 & 0.00337 & 0.00613 & 0.00168 & 0.00293 & 0.01067 & 0.01158 \\
\hline 6 & 373 & 0.97292 & 0.02708 & 0.00610 & 0.02098 & 0.03692 & 0.96308 & 0.18650 & 0.77658 & 0.00233 & 0.00788 & 0.00179 & 0.00152 & 0.01105 & 0.01123 \\
\hline 6.5 & 373 & 0.97120 & 0.02880 & 0.00469 & 0.02412 & 0.06039 & 0.93961 & 0.17907 & 0.76054 & 0.00270 & 0.00370 & 0.00159 & 0.00182 & 0.00719 & 0.00791 \\
\hline 8 & 373 & 0.96370 & 0.03630 & 0.00751 & 0.02879 & 0.05420 & 0.94580 & 0.18442 & 0.76138 & 0.00266 & 0.00381 & 0.00152 & 0.00214 & 0.00618 & 0.00642 \\
\hline 3 & 383 & 0.98719 & 0.01281 & 0.00090 & 0.01191 & 0.12783 & 0.87217 & 0.16892 & 0.70324 & 0.00247 & 0.01058 & 0.00137 & 0.00176 & 0.01497 & 0.01566 \\
\hline 5 & 383 & 0.97858 & 0.02142 & 0.00341 & 0.01802 & 0.10589 & 0.89411 & 0.17230 & 0.72181 & 0.00290 & 0.00662 & 0.00167 & 0.00226 & 0.01148 & 0.00969 \\
\hline 6 & 383 & 0.97361 & 0.02639 & 0.00441 & 0.02198 & 0.08547 & 0.91453 & 0.17702 & 0.73751 & 0.00227 & 0.00759 & 0.00178 & 0.00132 & 0.01217 & 0.01300 \\
\hline
\end{tabular}




\begin{tabular}{|c|c|c|c|c|c|c|c|c|c|c|c|c|c|c|c|}
\hline 6.5 & 383 & 0.97158 & 0.02842 & 0.00386 & 0.02456 & 0.08339 & 0.91661 & 0.17467 & 0.74194 & 0.00269 & 0.00310 & 0.00138 & 0.00216 & 0.00607 & 0.00635 \\
\hline 8 & 383 & 0.96358 & 0.03642 & 0.00658 & 0.02983 & 0.06905 & 0.93095 & 0.18041 & 0.75053 & 0.00253 & 0.00338 & 0.00190 & 0.00163 & 0.00740 & 0.00737 \\
\hline 3 & 393 & 0.98634 & 0.01366 & 0.00185 & 0.01181 & 0.17788 & 0.82212 & 0.16298 & 0.65913 & 0.00251 & 0.00949 & 0.00153 & 0.00208 & 0.01665 & 0.01494 \\
\hline 5 & 393 & 0.97705 & 0.02295 & 0.00370 & 0.01925 & 0.11428 & 0.88572 & 0.16739 & 0.71833 & 0.00255 & 0.00666 & 0.00142 & 0.00234 & 0.01204 & 0.01224 \\
\hline 6 & 393 & 0.97201 & 0.02799 & 0.00527 & 0.02272 & 0.12188 & 0.87812 & 0.17195 & 0.70616 & 0.00266 & 0.00577 & 0.00165 & 0.00195 & 0.00924 & 0.00886 \\
\hline 6.5 & 393 & 0.96965 & 0.03035 & 0.00533 & 0.02502 & 0.10963 & 0.89037 & 0.17150 & 0.71887 & 0.00219 & 0.00322 & 0.00133 & 0.00173 & 0.00594 & 0.00728 \\
\hline 8 & 393 & 0.96148 & 0.03852 & 0.00808 & 0.03044 & 0.10244 & 0.89756 & 0.17328 & 0.72428 & 0.00253 & 0.00400 & 0.00200 & 0.00164 & 0.00802 & 0.00753 \\
\hline 3 & 403 & 0.98700 & 0.01300 & 0.00149 & 0.01152 & 0.24762 & 0.75238 & 0.14716 & 0.60522 & 0.00209 & 0.01072 & 0.00123 & 0.00135 & 0.01654 & 0.01474 \\
\hline 5 & 403 & 0.97713 & 0.02287 & 0.00331 & 0.01957 & 0.16307 & 0.83693 & 0.16102 & 0.67591 & 0.00241 & 0.00579 & 0.00163 & 0.00160 & 0.00918 & 0.00891 \\
\hline 6 & 403 & 0.97244 & 0.02756 & 0.00390 & 0.02366 & 0.14623 & 0.85377 & 0.16652 & 0.68725 & 0.00242 & 0.00631 & 0.00202 & 0.00225 & 0.01132 & 0.01112 \\
\hline 6.5 & 403 & 0.96947 & 0.03053 & 0.00473 & 0.02581 & 0.13963 & 0.86037 & 0.16324 & 0.69713 & 0.00276 & 0.00391 & 0.00133 & 0.00235 & 0.00868 & 0.00879 \\
\hline 8 & 403 & 0.96095 & 0.03905 & 0.00699 & 0.03205 & 0.13312 & 0.86688 & 0.16633 & 0.70055 & 0.00341 & 0.00349 & 0.00221 & 0.00236 & 0.00641 & 0.00544 \\
\hline 3 & 413 & 0.98714 & 0.01286 & 0.00132 & 0.01154 & 0.34316 & 0.65684 & 0.12773 & 0.52911 & 0.00255 & 0.00982 & 0.00159 & 0.00186 & 0.01685 & 0.01467 \\
\hline 5 & 413 & 0.97657 & 0.02343 & 0.00415 & 0.01928 & 0.22806 & 0.77194 & 0.14977 & 0.62217 & 0.00314 & 0.00478 & 0.00199 & 0.00250 & 0.00784 & 0.00760 \\
\hline 6 & 413 & 0.96880 & 0.03120 & 0.00558 & 0.02562 & 0.19891 & 0.80109 & 0.15297 & 0.64812 & 0.00707 & 0.00632 & 0.00291 & 0.00552 & 0.00976 & 0.00952 \\
\hline 6.5 & 413 & 0.96813 & 0.03187 & 0.00541 & 0.02646 & 0.18612 & 0.81388 & 0.15609 & 0.65779 & 0.00195 & 0.00401 & 0.00167 & 0.00094 & 0.00845 & 0.00675 \\
\hline 8 & 413 & 0.96026 & 0.03974 & 0.00741 & 0.03233 & 0.17174 & 0.82826 & 0.16388 & 0.66438 & 0.00298 & 0.00430 & 0.00212 & 0.00204 & 0.00751 & 0.00726 \\
\hline 3 & 423 & 0.98449 & 0.01551 & 0.00364 & 0.01187 & 0.42490 & 0.57510 & 0.12214 & 0.45296 & 0.00368 & 0.01103 & 0.00237 & 0.00315 & 0.01388 & 0.01568 \\
\hline 5 & 423 & 0.97621 & 0.02379 & 0.00403 & 0.01976 & 0.28888 & 0.71112 & 0.13915 & 0.57196 & 0.00399 & 0.02014 & 0.00238 & 0.00321 & 0.00930 & 0.01915 \\
\hline 6 & 423 & 0.97075 & 0.02925 & 0.00479 & 0.02445 & 0.24485 & 0.75515 & 0.14801 & 0.60714 & 0.00378 & 0.00635 & 0.00185 & 0.00350 & 0.01033 & 0.00947 \\
\hline 6.5 & 423 & 0.96719 & 0.03281 & 0.00513 & 0.02768 & 0.24313 & 0.75687 & 0.14604 & 0.61083 & 0.00264 & 0.00492 & 0.00169 & 0.00190 & 0.00753 & 0.00598 \\
\hline 8 & 423 & 0.95821 & 0.04179 & 0.00783 & 0.03396 & 0.22187 & 0.77813 & 0.15377 & 0.62436 & 0.00327 & 0.00469 & 0.00187 & 0.00256 & 0.00715 & 0.00805 \\
\hline 3 & 433 & 0.98790 & 0.01210 & 0.00182 & 0.01027 & 0.53116 & 0.46884 & 0.09177 & 0.37707 & 0.00346 & 0.01087 & 0.00244 & 0.00258 & 0.01403 & 0.01199 \\
\hline 5 & 433 & 0.97712 & 0.02288 & 0.00431 & 0.01857 & 0.37224 & 0.62776 & 0.12555 & 0.50221 & 0.00403 & 0.01345 & 0.00242 & 0.00288 & 0.00730 & 0.01284 \\
\hline 6 & 433 & 0.96901 & 0.03099 & 0.00512 & 0.02587 & 0.31838 & 0.68162 & 0.13611 & 0.54551 & 0.00358 & 0.00629 & 0.00276 & 0.00262 & 0.00890 & 0.00702 \\
\hline 6.5 & 433 & 0.96620 & 0.03380 & 0.00515 & 0.02865 & 0.30497 & 0.69503 & 0.13191 & 0.56312 & 0.00339 & 0.00856 & 0.00187 & 0.00248 & 0.00614 & 0.00811 \\
\hline 8 & 433 & 0.95702 & 0.04298 & 0.00778 & 0.03520 & 0.27434 & 0.72566 & 0.14301 & 0.58266 & 0.00328 & 0.00476 & 0.00254 & 0.00209 & 0.00554 & 0.00709 \\
\hline 3 & 443 & 0.98795 & 0.01205 & 0.00218 & 0.00987 & 0.63795 & 0.36205 & 0.06947 & 0.29259 & 0.00563 & 0.01378 & 0.00345 & 0.00406 & 0.01212 & 0.00850 \\
\hline 5 & 443 & 0.97764 & 0.02236 & 0.00220 & 0.02015 & 0.45485 & 0.54515 & 0.11068 & 0.43447 & 0.00452 & 0.01480 & 0.00255 & 0.00345 & 0.01231 & 0.01421 \\
\hline 6 & 443 & 0.96871 & 0.03129 & 0.00423 & 0.02706 & 0.39601 & 0.60399 & 0.11912 & 0.48487 & 0.00647 & 0.01266 & 0.00368 & 0.00491 & 0.00946 & 0.01320 \\
\hline 6.5 & 443 & 0.96501 & 0.03499 & 0.00488 & 0.03010 & 0.37028 & 0.62972 & 0.12407 & 0.50565 & 0.00684 & 0.01595 & 0.00243 & 0.00514 & 0.00810 & 0.01365 \\
\hline 8 & 443 & 0.95474 & 0.04526 & 0.00822 & 0.03704 & 0.33465 & 0.66535 & 0.13153 & 0.53382 & 0.00306 & 0.00470 & 0.00266 & 0.00197 & 0.00703 & 0.00719 \\
\hline 3 & 453 & 0.98759 & 0.01241 & 0.00211 & 0.01031 & 0.75662 & 0.24338 & 0.04736 & 0.19602 & 0.00691 & 0.02074 & 0.00370 & 0.00484 & 0.01609 & 0.01378 \\
\hline 5 & 453 & 0.97775 & 0.02225 & 0.00365 & 0.01861 & 0.54911 & 0.45089 & 0.09169 & 0.35919 & 0.00351 & 0.01005 & 0.00227 & 0.00246 & 0.01143 & 0.01003 \\
\hline 6 & 453 & 0.96945 & 0.03055 & 0.00456 & 0.02599 & 0.48629 & 0.51371 & 0.09995 & 0.41376 & 0.00643 & 0.02184 & 0.00344 & 0.00444 & 0.00995 & 0.01824 \\
\hline 6.5 & 453 & 0.96184 & 0.03816 & 0.00645 & 0.03171 & 0.45487 & 0.54513 & 0.10477 & 0.44036 & 0.00476 & 0.00957 & 0.00208 & 0.00420 & 0.00797 & 0.00971 \\
\hline 8 & 453 & 0.95395 & 0.04605 & 0.00880 & 0.03725 & 0.39950 & 0.60050 & 0.12118 & 0.47932 & 0.00561 & 0.00438 & 0.00381 & 0.00428 & 0.00626 & 0.00508 \\
\hline 5 & 463 & 0.98138 & 0.01862 & 0.00154 & 0.01708 & 0.63897 & 0.36103 & 0.07530 & 0.28573 & 0.00483 & 0.01001 & 0.00283 & 0.00415 & 0.00937 & 0.00800 \\
\hline 6 & 463 & 0.96883 & 0.03117 & 0.00447 & 0.02670 & 0.56673 & 0.43327 & 0.08900 & 0.34427 & 0.00987 & 0.00615 & 0.00551 & 0.00712 & 0.00699 & 0.00479 \\
\hline 6.5 & 463 & 0.96880 & 0.03120 & 0.00303 & 0.02817 & 0.52715 & 0.47285 & 0.09118 & 0.38167 & 0.00266 & 0.02013 & 0.00160 & 0.00183 & 0.00859 & 0.01816 \\
\hline 8 & 463 & 0.95410 & 0.04590 & 0.00783 & 0.03807 & 0.48569 & 0.51431 & 0.10351 & 0.41081 & 0.00429 & 0.00577 & 0.00212 & 0.00386 & 0.00745 & 0.00801 \\
\hline 5 & 473 & 0.98194 & 0.01806 & 0.00276 & 0.01530 & 0.74766 & 0.25234 & 0.05028 & 0.20206 & 0.00942 & 0.00923 & 0.00572 & 0.00667 & 0.00784 & 0.00834 \\
\hline 6 & 473 & 0.97241 & 0.02759 & 0.00434 & 0.02325 & 0.66592 & 0.33408 & 0.06595 & 0.26813 & 0.00729 & 0.00725 & 0.00340 & 0.00519 & 0.00676 & 0.00540 \\
\hline 6.5 & 473 & 0.96764 & 0.03236 & 0.00483 & 0.02753 & 0.62868 & 0.37132 & 0.07380 & 0.29752 & 0.00672 & 0.00851 & 0.00376 & 0.00469 & 0.00728 & 0.00476 \\
\hline 8 & 473 & 0.95394 & 0.04606 & 0.00838 & 0.03768 & 0.55157 & 0.44843 & 0.08688 & 0.36155 & 0.00423 & 0.00937 & 0.00299 & 0.00382 & 0.00759 & 0.00768 \\
\hline
\end{tabular}

Table S6 - VLE data of the system ethanol/gas mix $2 ; u\left(x_{\text {Eth }}^{\mathrm{L}}\right)=u\left(x_{\text {gas }}^{\mathrm{L}}\right)$ and $u\left(x_{\mathrm{Eth}}^{\mathrm{v}}\right)=u\left(x_{\mathrm{gas}}^{\mathrm{v}}\right)$

\begin{tabular}{|c|c|c|c|c|c|c|c|c|c|c|c|c|c|c|c|}
\hline$p / \mathrm{MPa}$ & $T / \mathrm{K}$ & $x_{\text {Eth }}^{\mathrm{L}}$ & $x_{\text {gas }}^{\mathrm{L}}$ & $x_{\mathrm{O}_{2}}^{\mathrm{L}}$ & $x_{\mathrm{N}_{2}}^{\mathrm{L}}$ & $x_{\mathrm{Eth}}^{\mathrm{v}}$ & $x_{\text {gas }}^{v}$ & $x_{\mathrm{O}_{2}}^{v}$ & $x_{\mathrm{N}_{2}}^{v}$ & $u\left(x_{\mathrm{Eth}}^{\mathrm{L}}\right)$ & $u\left(x_{\mathrm{Eth}}^{\mathrm{v}}\right)$ & $u\left(x_{\mathrm{O}_{2}}^{\mathrm{L}}\right)$ & $u\left(x_{\mathrm{O}_{2}}^{v}\right)$ & $u\left(x_{\mathrm{N}_{2}}^{\mathrm{L}}\right)$ & $u\left(x_{N_{2}}^{v}\right)$ \\
\hline 3 & 303 & 0.98168 & 0.01832 & 0.01268 & 0.00565 & 0.00785 & 0.99215 & 0.74860 & 0.24355 & 0.00600 & 0.00966 & 0.00327 & 0.02149 & 0.00486 & 0.01988 \\
\hline 5 & 303 & 0.97071 & 0.02929 & 0.02421 & 0.00508 & 0.00322 & 0.99678 & 0.76946 & 0.22731 & 0.00400 & 0.01220 & 0.00336 & 0.01947 & 0.00185 & 0.01695 \\
\hline 6 & 303 & 0.96800 & 0.03200 & 0.02580 & 0.00620 & 0.00071 & 0.99929 & 0.76905 & 0.23023 & 0.00497 & 0.00528 & 0.00407 & 0.00853 & 0.00193 & 0.00880 \\
\hline 6.5 & 303 & 0.96490 & 0.03510 & 0.02812 & 0.00698 & 0.00721 & 0.99279 & 0.74478 & 0.24801 & 0.00544 & 0.00876 & 0.00461 & 0.01468 & 0.00268 & 0.01542 \\
\hline 8 & 303 & 0.95737 & 0.04263 & 0.03498 & 0.00765 & 0.00876 & 0.99124 & 0.76017 & 0.23107 & 0.00454 & 0.00750 & 0.00381 & 0.01736 & 0.00240 & 0.01738 \\
\hline 3 & 313 & 0.98369 & 0.01631 & 0.01102 & 0.00529 & 0.00683 & 0.99317 & 0.74953 & 0.24364 & 0.00539 & 0.00938 & 0.00410 & 0.01795 & 0.00300 & 0.01823 \\
\hline 5 & 313 & 0.97382 & 0.02618 & 0.02127 & 0.00491 & 0.00063 & 0.99937 & 0.77515 & 0.22421 & 0.00220 & 0.00937 & 0.00229 & 0.01712 & 0.00162 & 0.01704 \\
\hline 6 & 313 & 0.96664 & 0.03336 & 0.02717 & 0.00619 & 0.00269 & 0.99731 & 0.77043 & 0.22688 & 0.00395 & 0.00728 & 0.00298 & 0.01282 & 0.00205 & 0.01088 \\
\hline 6.5 & 313 & 0.96594 & 0.03406 & 0.02728 & 0.00678 & 0.01748 & 0.98252 & 0.74031 & 0.24222 & 0.00337 & 0.00870 & 0.00265 & 0.01416 & 0.00186 & 0.01292 \\
\hline 8 & 313 & 0.95625 & 0.04375 & 0.03598 & 0.00777 & 0.00935 & 0.99065 & 0.75358 & 0.23708 & 0.00392 & 0.00816 & 0.00293 & 0.01452 & 0.00168 & 0.01343 \\
\hline 3 & 323 & 0.98290 & 0.01710 & 0.01278 & 0.00432 & 0.01594 & 0.98406 & 0.74328 & 0.24078 & 0.00382 & 0.00750 & 0.00305 & 0.01130 & 0.00261 & 0.01078 \\
\hline 5 & 323 & 0.97322 & 0.02678 & 0.02181 & 0.00497 & 0.00151 & 0.99849 & 0.77390 & 0.22459 & 0.00330 & 0.01088 & 0.00246 & 0.01998 & 0.00189 & 0.01881 \\
\hline 6 & 323 & 0.96614 & 0.03386 & 0.02818 & 0.00567 & 0.00417 & 0.99583 & 0.76878 & 0.22705 & 0.00361 & 0.00907 & 0.00331 & 0.01993 & 0.00196 & 0.01793 \\
\hline 6.5 & 323 & 0.96333 & 0.03667 & 0.02979 & 0.00688 & 0.00690 & 0.99310 & 0.76543 & 0.22767 & 0.00359 & 0.00836 & 0.00235 & 0.01364 & 0.00251 & 0.01257 \\
\hline 8 & 323 & 0.95766 & 0.04234 & 0.03478 & 0.00756 & 0.01193 & 0.98807 & 0.75473 & 0.23334 & 0.00283 & 0.00808 & 0.00219 & 0.01970 & 0.00202 & 0.01727 \\
\hline 3 & 333 & 0.98215 & 0.01785 & 0.01353 & 0.00432 & 0.02173 & 0.97827 & 0.73938 & 0.23889 & 0.00306 & 0.00725 & 0.00316 & 0.01527 & 0.00298 & 0.01587 \\
\hline 5 & 333 & 0.97190 & 0.02810 & 0.02335 & 0.00475 & 0.00039 & 0.99961 & 0.78046 & 0.21915 & 0.00335 & 0.01091 & 0.00268 & 0.02166 & 0.00201 & 0.02118 \\
\hline 6 & 333 & 0.96452 & 0.03548 & 0.02954 & 0.00595 & 0.00594 & 0.99406 & 0.76700 & 0.22707 & 0.00337 & 0.00887 & 0.00341 & 0.01178 & 0.00189 & 0.01258 \\
\hline 6.5 & 333 & 0.96200 & 0.03800 & 0.03147 & 0.00653 & 0.00314 & 0.99686 & 0.76636 & 0.23050 & 0.00359 & 0.00547 & 0.00244 & 0.01452 & 0.00204 & 0.01371 \\
\hline 8 & 333 & 0.95515 & 0.04485 & 0.03736 & 0.00749 & 0.00198 & 0.99802 & 0.76638 & 0.23164 & 0.00393 & 0.01011 & 0.00255 & 0.02421 & 0.00220 & 0.02419 \\
\hline 3 & 343 & 0.98216 & 0.01784 & 0.01328 & 0.00456 & 0.03911 & 0.96089 & 0.72552 & 0.23536 & 0.00409 & 0.01123 & 0.00354 & 0.01444 & 0.00255 & 0.01310 \\
\hline 5 & 343 & 0.97101 & 0.02899 & 0.02401 & 0.00498 & 0.00787 & 0.99213 & 0.76665 & 0.22548 & 0.00302 & 0.01020 & 0.00209 & 0.01899 & 0.00162 & 0.01808 \\
\hline 6 & 343 & 0.96506 & 0.03494 & 0.02904 & 0.00589 & 0.01732 & 0.98268 & 0.75882 & 0.22386 & 0.00303 & 0.00477 & 0.00228 & 0.01107 & 0.00174 & 0.01020 \\
\hline 6.5 & 343 & 0.96353 & 0.03647 & 0.02996 & 0.00651 & 0.00384 & 0.99616 & 0.76567 & 0.23048 & 0.00324 & 0.00807 & 0.00224 & 0.01214 & 0.00193 & 0.01026 \\
\hline 8 & 343 & 0.95594 & 0.04406 & 0.03616 & 0.00791 & 0.00908 & 0.99092 & 0.76343 & 0.22749 & 0.00374 & 0.01024 & 0.00315 & 0.02439 & 0.00249 & 0.02400 \\
\hline 3 & 353 & 0.98196 & 0.01804 & 0.01368 & 0.00437 & 0.05628 & 0.94372 & 0.71219 & 0.23153 & 0.00376 & 0.01446 & 0.00266 & 0.02262 & 0.00228 & 0.01749 \\
\hline 5 & 353 & 0.97108 & 0.02892 & 0.02367 & 0.00525 & 0.02166 & 0.97834 & 0.75630 & 0.22204 & 0.00300 & 0.00752 & 0.00248 & 0.01614 & 0.00168 & 0.01574 \\
\hline 6 & 353 & 0.96563 & 0.03437 & 0.02839 & 0.00598 & 0.02847 & 0.97153 & 0.74779 & 0.22374 & 0.00297 & 0.00577 & 0.00271 & 0.01145 & 0.00138 & 0.01129 \\
\hline
\end{tabular}




\begin{tabular}{|c|c|c|c|c|c|c|c|c|c|c|c|c|c|c|c|}
\hline 6.5 & 353 & 0.96231 & 0.03769 & 0.03097 & 0.00672 & 0.02426 & 0.97574 & 0.74830 & 0.22744 & 0.00310 & 0.00824 & 0.00263 & 0.01658 & 0.00186 & 0.01492 \\
\hline 8 & 353 & 0.95455 & 0.04545 & 0.03637 & 0.00908 & 0.00999 & 0.99001 & 0.76116 & 0.22885 & 0.00441 & 0.00955 & 0.00346 & 0.02006 & 0.00269 & 0.02083 \\
\hline 3 & 363 & 0.98450 & 0.01550 & 0.01087 & 0.00463 & 0.05527 & 0.94473 & 0.70853 & 0.23621 & 0.00407 & 0.01049 & 0.00313 & 0.02040 & 0.00253 & 0.01746 \\
\hline 5 & 363 & 0.97246 & 0.02754 & 0.02199 & 0.00555 & 0.03347 & 0.96653 & 0.75773 & 0.20881 & 0.00255 & 0.00854 & 0.00213 & 0.01647 & 0.00161 & 0.01621 \\
\hline 6 & 363 & 0.96765 & 0.03235 & 0.02674 & 0.00560 & 0.04441 & 0.95559 & 0.73364 & 0.22195 & 0.00264 & 0.00639 & 0.00240 & 0.01384 & 0.00130 & 0.01509 \\
\hline 6.5 & 363 & 0.96367 & 0.03633 & 0.02915 & 0.00718 & 0.03410 & 0.96590 & 0.72941 & 0.23649 & 0.00412 & 0.00989 & 0.00283 & 0.01744 & 0.00250 & 0.01624 \\
\hline 8 & 363 & 0.95520 & 0.04480 & 0.03596 & 0.00884 & 0.00892 & 0.99108 & 0.75795 & 0.23313 & 0.00444 & 0.01328 & 0.00343 & 0.02550 & 0.00293 & 0.02017 \\
\hline 3 & 373 & 0.98451 & 0.01549 & 0.01132 & 0.00417 & 0.09590 & 0.90410 & 0.68525 & 0.21886 & 0.00334 & 0.01094 & 0.00273 & 0.01611 & 0.00222 & 0.01761 \\
\hline 5 & 373 & 0.97232 & 0.02768 & 0.02248 & 0.00521 & 0.05567 & 0.94433 & 0.73054 & 0.21379 & 0.00231 & 0.00702 & 0.00197 & 0.01182 & 0.00136 & 0.01076 \\
\hline 6 & 373 & 0.96570 & 0.03430 & 0.02769 & 0.00662 & 0.05990 & 0.94010 & 0.72592 & 0.21418 & 0.00301 & 0.00747 & 0.00247 & 0.01233 & 0.00185 & 0.01307 \\
\hline 6.5 & 373 & 0.96259 & 0.03741 & 0.03029 & 0.00712 & 0.05877 & 0.94123 & 0.71034 & 0.23089 & 0.00307 & 0.01048 & 0.00220 & 0.02082 & 0.00204 & 0.02275 \\
\hline 8 & 373 & 0.95362 & 0.04638 & 0.03727 & 0.00912 & 0.04842 & 0.95158 & 0.74510 & 0.20648 & 0.00435 & 0.01701 & 0.00343 & 0.03204 & 0.00205 & 0.02903 \\
\hline 3 & 383 & 0.98693 & 0.01307 & 0.00951 & 0.00355 & 0.14009 & 0.85991 & 0.64853 & 0.21138 & 0.00390 & 0.00957 & 0.00367 & 0.01712 & 0.00204 & 0.01964 \\
\hline 5 & 383 & 0.97254 & 0.02746 & 0.02180 & 0.00566 & 0.09611 & 0.90389 & 0.69841 & 0.20548 & 0.00328 & 0.00730 & 0.00237 & 0.01469 & 0.00157 & 0.01435 \\
\hline 6 & 383 & 0.96679 & 0.03321 & 0.02619 & 0.00702 & 0.07061 & 0.92939 & 0.71746 & 0.21193 & 0.00305 & 0.00647 & 0.00264 & 0.01379 & 0.00189 & 0.01426 \\
\hline 6.5 & 383 & 0.96098 & 0.03902 & 0.03108 & 0.00794 & 0.05993 & 0.94007 & 0.72624 & 0.21383 & 0.00314 & 0.00778 & 0.00261 & 0.01381 & 0.00138 & 0.01080 \\
\hline 8 & 383 & 0.95504 & 0.04496 & 0.03672 & 0.00824 & 0.03978 & 0.96022 & 0.74829 & 0.21193 & 0.00396 & 0.01574 & 0.00290 & 0.03091 & 0.00249 & 0.03072 \\
\hline 3 & 393 & 0.98453 & 0.01547 & 0.01100 & 0.00447 & 0.16549 & 0.83451 & 0.62445 & 0.21006 & 0.00394 & 0.00841 & 0.00296 & 0.01746 & 0.00236 & 0.01668 \\
\hline 5 & 393 & 0.97134 & 0.02866 & 0.02377 & 0.00488 & 0.11605 & 0.88395 & 0.68219 & 0.20176 & 0.00306 & 0.00748 & 0.00278 & 0.01156 & 0.00148 & 0.01286 \\
\hline 6 & 393 & 0.96589 & 0.03411 & 0.02830 & 0.00582 & 0.11641 & 0.88359 & 0.67957 & 0.20402 & 0.00337 & 0.00766 & 0.00277 & 0.01398 & 0.00188 & 0.01509 \\
\hline 6.5 & 393 & 0.96154 & 0.03846 & 0.03108 & 0.00738 & 0.10825 & 0.89175 & 0.67742 & 0.21433 & 0.00253 & 0.01020 & 0.00279 & 0.01873 & 0.00175 & 0.01985 \\
\hline 8 & 393 & 0.95219 & 0.04781 & 0.03874 & 0.00907 & 0.05983 & 0.94017 & 0.72765 & 0.21252 & 0.00745 & 0.01787 & 0.00530 & 0.03666 & 0.00363 & 0.03442 \\
\hline 3 & 403 & 0.98579 & 0.01421 & 0.00985 & 0.00436 & 0.24954 & 0.75046 & 0.56498 & 0.18548 & 0.00538 & 0.00968 & 0.00315 & 0.01689 & 0.00467 & 0.01592 \\
\hline 5 & 403 & 0.97188 & 0.02812 & 0.02196 & 0.00615 & 0.15852 & 0.84148 & 0.65079 & 0.19069 & 0.00632 & 0.00931 & 0.00485 & 0.01459 & 0.00221 & 0.01168 \\
\hline 6 & 403 & 0.96492 & 0.03508 & 0.02858 & 0.00650 & 0.15992 & 0.84008 & 0.64636 & 0.19371 & 0.00361 & 0.00820 & 0.00281 & 0.01332 & 0.00187 & 0.01664 \\
\hline 6.5 & 403 & 0.96277 & 0.03723 & 0.02992 & 0.00731 & 0.13201 & 0.86799 & 0.65478 & 0.21321 & 0.00291 & 0.01112 & 0.00220 & 0.01848 & 0.00228 & 0.01936 \\
\hline 8 & 403 & 0.95184 & 0.04816 & 0.03821 & 0.00995 & 0.14104 & 0.85896 & 0.66395 & 0.19501 & 0.00359 & 0.00896 & 0.00303 & 0.01420 & 0.00181 & 0.01701 \\
\hline 3 & 413 & 0.98635 & 0.01365 & 0.00826 & 0.00539 & 0.33248 & 0.66752 & 0.49644 & 0.17109 & 0.00522 & 0.01224 & 0.00363 & 0.01469 & 0.00298 & 0.01485 \\
\hline 5 & 413 & 0.97201 & 0.02799 & 0.02233 & 0.00566 & 0.22256 & 0.77744 & 0.60673 & 0.17070 & 0.00367 & 0.01379 & 0.00262 & 0.02009 & 0.00176 & 0.02147 \\
\hline 6 & 413 & 0.96545 & 0.03455 & 0.02789 & 0.00666 & 0.19817 & 0.80183 & 0.62122 & 0.18062 & 0.00365 & 0.00874 & 0.00271 & 0.01280 & 0.00239 & 0.01212 \\
\hline 6.5 & 413 & 0.96256 & 0.03744 & 0.03067 & 0.00678 & 0.18580 & 0.81420 & 0.61913 & 0.19507 & 0.00343 & 0.01129 & 0.00270 & 0.01839 & 0.00235 & 0.01927 \\
\hline 8 & 413 & 0.95035 & 0.04965 & 0.03944 & 0.01021 & 0.15369 & 0.84631 & 0.65740 & 0.18890 & 0.00477 & 0.00838 & 0.00315 & 0.01682 & 0.00287 & 0.01798 \\
\hline 3 & 423 & 0.98703 & 0.01297 & 0.00829 & 0.00467 & 0.43357 & 0.56643 & 0.42456 & 0.14187 & 0.00423 & 0.00992 & 0.00318 & 0.01517 & 0.00304 & 0.01255 \\
\hline 5 & 423 & 0.97252 & 0.02748 & 0.02179 & 0.00569 & 0.27463 & 0.72537 & 0.56030 & 0.16507 & 0.00379 & 0.01750 & 0.00360 & 0.02239 & 0.00130 & 0.02287 \\
\hline 6 & 423 & 0.96673 & 0.03327 & 0.02653 & 0.00674 & 0.24909 & 0.75091 & 0.58256 & 0.16836 & 0.00391 & 0.00767 & 0.00319 & 0.01276 & 0.00176 & 0.01370 \\
\hline 6.5 & 423 & 0.96006 & 0.03994 & 0.03168 & 0.00826 & 0.22660 & 0.77340 & 0.57461 & 0.19879 & 0.00378 & 0.01225 & 0.00266 & 0.01838 & 0.00250 & 0.01936 \\
\hline 8 & 423 & 0.94776 & 0.05224 & 0.04225 & 0.00999 & 0.19565 & 0.80435 & 0.61938 & 0.18497 & 0.00414 & 0.00863 & 0.00357 & 0.02182 & 0.00225 & 0.02082 \\
\hline 3 & 433 & 0.98777 & 0.01223 & 0.00663 & 0.00560 & 0.54941 & 0.45059 & 0.33498 & 0.11561 & 0.00541 & 0.03310 & 0.00472 & 0.02340 & 0.00545 & 0.01372 \\
\hline 5 & 433 & 0.97480 & 0.02520 & 0.02039 & 0.00481 & 0.34370 & 0.65630 & 0.51141 & 0.14489 & 0.00467 & 0.01621 & 0.00364 & 0.01664 & 0.00262 & 0.02049 \\
\hline 6 & 433 & 0.96471 & 0.03529 & 0.02845 & 0.00683 & 0.31087 & 0.68913 & 0.52587 & 0.16326 & 0.00489 & 0.01448 & 0.00372 & 0.01346 & 0.00247 & 0.01644 \\
\hline 6.5 & 433 & 0.95870 & 0.04130 & 0.03250 & 0.00880 & 0.30719 & 0.69281 & 0.53810 & 0.15470 & 0.00409 & 0.01184 & 0.00330 & 0.01326 & 0.00186 & 0.01709 \\
\hline 8 & 433 & 0.94870 & 0.05130 & 0.04124 & 0.01006 & 0.26412 & 0.73588 & 0.55803 & 0.17786 & 0.00362 & 0.01401 & 0.00331 & 0.01552 & 0.00149 & 0.01951 \\
\hline 3 & 443 & 0.98183 & 0.01817 & 0.00576 & 0.01241 & 0.65690 & 0.34310 & 0.24991 & 0.09319 & 0.01164 & 0.02749 & 0.00845 & 0.02011 & 0.00887 & 0.01260 \\
\hline 5 & 443 & 0.97391 & 0.02609 & 0.02111 & 0.00498 & 0.42370 & 0.57630 & 0.44210 & 0.13420 & 0.00694 & 0.03030 & 0.00591 & 0.03178 & 0.00338 & 0.03985 \\
\hline 6 & 443 & 0.96678 & 0.03322 & 0.02508 & 0.00815 & 0.38085 & 0.61915 & 0.49992 & 0.11923 & 0.00397 & 0.01910 & 0.00274 & 0.01939 & 0.00220 & 0.02446 \\
\hline 6.5 & 443 & 0.95741 & 0.04259 & 0.03252 & 0.01007 & 0.31670 & 0.68330 & 0.52181 & 0.16149 & 0.00674 & 0.02157 & 0.00564 & 0.02814 & 0.00247 & 0.03120 \\
\hline 8 & 443 & 0.94837 & 0.05163 & 0.04138 & 0.01025 & 0.28871 & 0.71129 & 0.55434 & 0.15695 & 0.00305 & 0.01058 & 0.00240 & 0.01452 & 0.00147 & 0.01662 \\
\hline 5 & 453 & 0.97041 & 0.02959 & 0.02246 & 0.00713 & 0.49529 & 0.50471 & 0.40326 & 0.10145 & 0.01398 & 0.02505 & 0.01122 & 0.02477 & 0.00553 & 0.02924 \\
\hline 6 & 453 & 0.96544 & 0.03456 & 0.02664 & 0.00792 & 0.45796 & 0.54204 & 0.40192 & 0.14012 & 0.00414 & 0.03597 & 0.00434 & 0.03445 & 0.00246 & 0.02984 \\
\hline 6.5 & 453 & 0.95969 & 0.04031 & 0.03174 & 0.00858 & 0.45578 & 0.54422 & 0.41630 & 0.12791 & 0.00390 & 0.01427 & 0.00315 & 0.01390 & 0.00212 & 0.01980 \\
\hline 8 & 453 & 0.94407 & 0.05593 & 0.04511 & 0.01082 & 0.36423 & 0.63577 & 0.48998 & 0.14579 & 0.00559 & 0.01444 & 0.00464 & 0.01110 & 0.00216 & 0.01447 \\
\hline 5 & 463 & 0.97409 & 0.02591 & 0.01895 & 0.00696 & 0.61710 & 0.38290 & 0.29227 & 0.09063 & 0.00817 & 0.02203 & 0.00634 & 0.01728 & 0.00411 & 0.01855 \\
\hline 6 & 463 & 0.96709 & 0.03291 & 0.02538 & 0.00753 & 0.54324 & 0.45676 & 0.33789 & 0.11886 & 0.00460 & 0.02584 & 0.00376 & 0.03250 & 0.00274 & 0.03395 \\
\hline 6.5 & 463 & 0.96151 & 0.03849 & 0.02963 & 0.00886 & 0.52896 & 0.47104 & 0.35749 & 0.11355 & 0.00981 & 0.01436 & 0.00675 & 0.01134 & 0.00700 & 0.01616 \\
\hline 8 & 463 & 0.93891 & 0.06109 & 0.04856 & 0.01254 & 0.44440 & 0.55560 & 0.42981 & 0.12579 & 0.00537 & 0.01138 & 0.00478 & 0.01215 & 0.00252 & 0.01321 \\
\hline 6 & 473 & 0.97025 & 0.02975 & 0.02294 & 0.00680 & 0.65133 & 0.34867 & 0.25565 & 0.09302 & 0.00733 & 0.03442 & 0.00639 & 0.03340 & 0.00420 & 0.03076 \\
\hline 6.5 & 473 & 0.96371 & 0.03629 & 0.02411 & 0.01218 & 0.60993 & 0.39007 & 0.29397 & 0.09611 & 0.02077 & 0.03474 & 0.01169 & 0.02803 & 0.01242 & 0.01942 \\
\hline 8 & 473 & 0.94416 & 0.05584 & 0.04403 & 0.01181 & 0.52696 & 0.47304 & 0.36993 & 0.10311 & 0.00555 & 0.02427 & 0.00424 & 0.02001 & 0.00313 & 0.01190 \\
\hline
\end{tabular}

Table S7 - VLE data of the system ethanol/nitrogen of Klima et al. ${ }^{3}$, needed for the Peng-Robinson-EOS and illustration of ternary VLE data; the temperatures highlighted in red color were considered to be equal to the liquid phase experimental run, since the temperature of the vapor phase measurement at these points are given as $0.5 \mathrm{~K}$ below the liquid phase setpoints

\begin{tabular}{cccccc}
$p / \mathrm{MPa}$ & $T / \mathrm{K}$ & $x_{\text {Eth }}^{\mathrm{L}}$ & $x_{\mathrm{N}_{2}}^{\mathrm{L}}$ & $x_{\mathrm{Eth}}^{\mathrm{v}}$ & $x_{\mathrm{N}_{2}}^{\mathrm{v}}$ \\
\hline 3 & 303 & 0.98758 & 0.01242 & 0.00848 & 0.99152 \\
5 & 303 & 0.98204 & 0.01796 & 0.02302 & 0.97698 \\
6.5 & 303 & 0.98064 & 0.01936 & 0.01429 & 0.98571 \\
8 & 303 & 0.97355 & 0.02645 & 0.01106 & 0.98894 \\
3 & 313 & 0.98896 & 0.01104 & 0.02962 & 0.97038 \\
5 & 313 & 0.98139 & 0.01861 & 0.02640 & 0.97360 \\
6.5 & 313 & 0.97977 & 0.02023 & 0.01548 & 0.98452 \\
8 & 313 & 0.97391 & 0.02609 & 0.00634 & 0.99366 \\
3 & 323 & 0.99031 & 0.00969 & 0.01871 & 0.98129 \\
5 & 323 & 0.98118 & 0.01882 & 0.02793 & 0.97207 \\
6.5 & 323 & 0.97870 & 0.02130 & 0.02159 & 0.97841 \\
8 & 323 & 0.97318 & 0.02682 & 0.00905 & 0.99095 \\
3 & 333 & 0.98839 & 0.01161 & 0.03546 & 0.96454
\end{tabular}




\begin{tabular}{cccccc}
6.5 & 333 & 0.97818 & 0.02182 & 0.02904 & 0.97096 \\
8 & 333 & 0.97218 & 0.02782 & 0.00660 & 0.99340 \\
3 & 343 & 0.98876 & 0.01124 & 0.05482 & 0.94518 \\
6.5 & 343 & 0.97813 & 0.02187 & 0.03397 & 0.96603 \\
8 & 343 & 0.97162 & 0.02838 & 0.01129 & 0.98871 \\
3 & 353 & 0.98691 & 0.01309 & 0.04855 & 0.95145 \\
6.5 & 353 & 0.97736 & 0.02264 & 0.04806 & 0.95194 \\
8 & 353 & 0.97069 & 0.02931 & 0.02802 & 0.97198 \\
3 & 363 & 0.98920 & 0.01080 & 0.08379 & 0.91621 \\
6.5 & 363 & 0.97716 & 0.02284 & 0.05429 & 0.94571 \\
8 & 363 & 0.96987 & 0.03013 & 0.03839 & 0.96161 \\
3 & 373 & 0.98647 & 0.01353 & 0.10989 & 0.89011 \\
6.5 & 373 & 0.97575 & 0.02425 & 0.07708 & 0.92292 \\
8 & 373 & 0.96904 & 0.03096 & 0.06804 & 0.93196 \\
3 & 383 & 0.98719 & 0.01281 & 0.14136 & 0.85864 \\
6.5 & 383 & 0.97498 & 0.02502 & 0.09461 & 0.90539 \\
8 & 383 & 0.96718 & 0.03282 & 0.06379 & 0.93621 \\
3 & 393 & 0.98892 & 0.01108 & 0.18354 & 0.81646 \\
6.5 & 393 & 0.97488 & 0.02512 & 0.12380 & 0.87620 \\
8 & 393 & 0.96600 & 0.03400 & 0.11057 & 0.88943 \\
3 & 403 & 0.98544 & 0.01456 & 0.26644 & 0.73356 \\
6.5 & 403 & 0.97358 & 0.02642 & 0.15367 & 0.84633 \\
8 & 403 & 0.96532 & 0.03468 & 0.13375 & 0.86625 \\
3 & 413 & 0.98707 & 0.01293 & 0.35384 & 0.64616 \\
6.5 & 413 & 0.97311 & 0.02689 & 0.19941 & 0.80059 \\
8 & 413 & 0.96435 & 0.03565 & 0.16906 & 0.83094 \\
3 & 423 & 0.98656 & 0.01344 & 0.43989 & 0.56011 \\
5 & 423 & 0.97669 & 0.02331 & 0.30057 & 0.69943 \\
6.5 & 423 & 0.97209 & 0.02791 & 0.25594 & 0.74406 \\
8 & 423 & 0.96316 & 0.03684 & 0.20441 & 0.79559 \\
3 & 433 & 0.98844 & 0.01156 & 0.55030 & 0.44970 \\
5 & 433 & 0.97627 & 0.02373 & 0.38524 & 0.61476 \\
6.5 & 433 & 0.97193 & 0.02807 & 0.31734 & 0.68266 \\
8 & 433 & 0.96145 & 0.03855 & 0.26763 & 0.73237 \\
3 & 443 & 0.97950 & 0.02050 & 0.66965 & 0.33035 \\
5 & 443 & 0.97673 & 0.02327 & 0.46728 & 0.53272 \\
6.5 & 443 & 0.97190 & 0.02810 & 0.38137 & 0.61863 \\
8 & 443 & 0.96071 & 0.03929 & 0.33449 & 0.66551 \\
3 & 453 & 0.99066 & 0.00934 & 0.80752 & 0.19248 \\
5 & 453 & 0.97323 & 0.02677 & 0.56079 & 0.43921 \\
6.5 & 453 & 0.97158 & 0.02842 & 0.46514 & 0.53486 \\
8 & 453 & 0.96043 & 0.03957 & 0.40776 & 0.59224 \\
5 & 463 & 0.97867 & 0.02133 & 0.67169 & 0.32831 \\
6.5 & 463 & 0.97073 & 0.02927 & 0.55953 & 0.44047 \\
8 & 463 & 0.95934 & 0.04066 & 0.49059 & 0.50941 \\
5 & 473 & 0.98278 & 0.01722 & 0.76326 & 0.23674 \\
6.5 & 473 & 0.97563 & 0.02437 & 0.66346 & 0.33654 \\
8 & 473 & 0.96115 & 0.03885 & 0.58115 & 0.41885 \\
\hline & & & & & \\
& & & & & \\
\hline & &
\end{tabular}

Table S8 - ternary VLE data (consisting of Tables S4, S5, S6 and S7); compare Table S7 for temperatures highlighted in red color

\begin{tabular}{|c|c|c|c|c|c|c|c|c|}
\hline$p / \mathrm{MPa}$ & $T / \mathrm{K}$ & $x_{\mathrm{Eth}}^{\mathrm{L}}$ & $x_{\mathrm{Eth}}^{v}$ & $x_{\mathrm{O}_{2}}^{\mathrm{L}}$ & $x_{0_{2}}^{v}$ & $x_{\mathrm{N}_{2}}^{\mathrm{L}}$ & $x_{\mathrm{N}_{2}}^{\mathrm{v}}$ & system \\
\hline 3 & 303 & 0.98758 & 0.00848 & 0.00000 & 0.00000 & 0.01242 & 0.99152 & ethanol/nitrogen \\
\hline 3 & 303 & 0.98744 & 0.00264 & 0.00236 & 0.19499 & 0.01020 & 0.80237 & ethanol/gas mix 1 \\
\hline 3 & 303 & 0.98168 & 0.00785 & 0.01268 & 0.74860 & 0.00565 & 0.24355 & ethanol/gas mix 2 \\
\hline 3 & 303 & 0.98305 & 0.00087 & 0.01695 & 0.99913 & 0.00000 & 0.00000 & ethanol/oxygen \\
\hline 3 & 313 & 0.98896 & 0.02962 & 0.00000 & 0.00000 & 0.01104 & 0.97038 & ethanol/nitrogen \\
\hline 3 & 313 & 0.98722 & 0.00457 & 0.00245 & 0.18342 & 0.01033 & 0.81201 & ethanol/gas mix 1 \\
\hline 3 & 313 & 0.98369 & 0.00683 & 0.01102 & 0.74953 & 0.00529 & 0.24364 & ethanol/gas mix 2 \\
\hline 3 & 313 & 0.98319 & 0.00165 & 0.01681 & 0.99835 & 0.00000 & 0.00000 & ethanol/oxygen \\
\hline 3 & 323 & 0.99031 & 0.01871 & 0.00000 & 0.00000 & 0.00969 & 0.98129 & ethanol/nitrogen \\
\hline 3 & 323 & 0.98664 & 0.00767 & 0.00300 & 0.18512 & 0.01036 & 0.80720 & ethanol/gas mix 1 \\
\hline 3 & 323 & 0.98290 & 0.01594 & 0.01278 & 0.74328 & 0.00432 & 0.24078 & ethanol/gas mix 2 \\
\hline 3 & 323 & 0.98259 & 0.00238 & 0.01741 & 0.99762 & 0.00000 & 0.00000 & ethanol/oxygen \\
\hline 3 & 333 & 0.98839 & 0.03546 & 0.00000 & 0.00000 & 0.01161 & 0.96454 & ethanol/nitrogen \\
\hline 3 & 333 & 0.98345 & 0.00742 & 0.00377 & 0.18443 & 0.01278 & 0.80815 & ethanol/gas mix 1 \\
\hline 3 & 333 & 0.98215 & 0.02173 & 0.01353 & 0.73938 & 0.00432 & 0.23889 & ethanol/gas mix 2 \\
\hline 3 & 333 & 0.98056 & 0.00138 & 0.01944 & 0.99862 & 0.00000 & 0.00000 & ethanol/oxygen \\
\hline 3 & 343 & 0.98876 & 0.05482 & 0.00000 & 0.00000 & 0.01124 & 0.94518 & ethanol/nitrogen \\
\hline 3 & 343 & 0.98150 & 0.02512 & 0.00484 & 0.17898 & 0.01366 & 0.79590 & ethanol/gas mix 1 \\
\hline 3 & 343 & 0.98216 & 0.03911 & 0.01328 & 0.72552 & 0.00456 & 0.23536 & ethanol/gas mix 2 \\
\hline 3 & 343 & 0.98361 & 0.01643 & 0.01639 & 0.98357 & 0.00000 & 0.00000 & ethanol/oxygen \\
\hline 3 & 353 & 0.98691 & 0.04855 & 0.00000 & 0.00000 & 0.01309 & 0.95145 & ethanol/nitrogen \\
\hline 3 & 353 & 0.98226 & 0.04272 & 0.00514 & 0.18282 & 0.01260 & 0.77446 & ethanol/gas mix 1 \\
\hline 3 & 353 & 0.98196 & 0.05628 & 0.01368 & 0.71219 & 0.00437 & 0.23153 & ethanol/gas mix 2 \\
\hline 3 & 353 & 0.98272 & 0.02049 & 0.01728 & 0.97951 & 0.00000 & 0.00000 & ethanol/oxygen \\
\hline 3 & 363 & 0.98920 & 0.08379 & 0.00000 & 0.00000 & 0.01080 & 0.91621 & ethanol/nitrogen \\
\hline
\end{tabular}




\begin{tabular}{|c|c|c|c|c|c|c|c|}
\hline 363 & 0.98619 & 0.05254 & 0.00286 & 0.18787 & 0.01095 & 0.75958 & ethanol/gas mix 1 \\
\hline 363 & 0.98450 & 0.05527 & 0.01087 & 0.70853 & 0.00463 & 0.23621 & ethanol/gas mix 2 \\
\hline 363 & 0.98717 & 0.03695 & 0.01283 & 0.96305 & 0.00000 & 0.00000 & ethanol/oxygen \\
\hline 373 & 0.98647 & 0.10989 & 0.00000 & 0.00000 & 0.01353 & 0.89011 & ethanol/nitrogen \\
\hline 373 & 0.98534 & 0.06601 & 0.00187 & 0.18089 & 0.01279 & 0.75309 & ethanol/gas mix 1 \\
\hline 373 & 0.98451 & 0.09590 & 0.01132 & 0.68525 & 0.00417 & 0.21886 & ethanol/gas mix 2 \\
\hline 373 & 0.98373 & 0.10973 & 0.01627 & 0.89027 & 0.00000 & 0.00000 & ethanol/oxygen \\
\hline 383 & 0.98719 & 0.14136 & 0.00000 & 0.00000 & 0.01281 & 0.85864 & ethanol/nitrogen \\
\hline 383 & 0.98719 & 0.12783 & 0.00090 & 0.16892 & 0.01191 & 0.70324 & ethanol/gas mix 1 \\
\hline 383 & 0.98693 & 0.14009 & 0.00951 & 0.64853 & 0.00355 & 0.21138 & ethanol/gas mix 2 \\
\hline 383 & 0.98572 & 0.15958 & 0.01428 & 0.84042 & 0.00000 & 0.00000 & ethanol/oxygen \\
\hline 393 & 0.98892 & 0.18354 & 0.00000 & 0.00000 & 0.01108 & 0.81646 & ethanol/nitrogen \\
\hline 393 & 0.98634 & 0.17788 & 0.00185 & 0.16298 & 0.01181 & 0.65913 & ethanol/gas mix 1 \\
\hline 393 & 0.98453 & 0.16549 & 0.01100 & 0.62445 & 0.00447 & 0.21006 & ethanol/gas mix 2 \\
\hline 393 & 0.98524 & 0.21176 & 0.01476 & 0.78824 & 0.00000 & 0.00000 & ethanol/oxygen \\
\hline 403 & 0.98544 & 0.26644 & 0.00000 & 0.00000 & 0.01456 & 0.73356 & ethanol/nitrogen \\
\hline 403 & 0.98700 & 0.24762 & 0.00149 & 0.14716 & 0.01152 & 0.60522 & ethanol/gas mix 1 \\
\hline 403 & 0.98579 & 0.24954 & 0.00985 & 0.56498 & 0.00436 & 0.18548 & ethanol/gas mix 2 \\
\hline 403 & 0.98639 & 0.23464 & 0.01361 & 0.76536 & 0.00000 & 0.00000 & ethanol/oxygen \\
\hline 413 & 0.98707 & 0.35384 & 0.00000 & 0.00000 & 0.01293 & 0.64616 & ethanol/nitrogen \\
\hline 413 & 0.98714 & 0.34316 & 0.00132 & 0.12773 & 0.01154 & 0.52911 & ethanol/gas mix 1 \\
\hline 413 & 0.98635 & 0.33248 & 0.00826 & 0.49644 & 0.00539 & 0.17109 & ethanol/gas mix 2 \\
\hline 413 & 0.98714 & 0.34656 & 0.01286 & 0.65344 & 0.00000 & 0.00000 & ethanol/oxygen \\
\hline 423 & 0.98656 & 0.43989 & 0.00000 & 0.00000 & 0.01344 & 0.56011 & ethanol/nitrogen \\
\hline 423 & 0.98449 & 0.42490 & 0.00364 & 0.12214 & 0.01187 & 0.45296 & ethanol/gas mix 1 \\
\hline 423 & 0.98703 & 0.43357 & 0.00829 & 0.42456 & 0.00467 & 0.14187 & ethanol/gas mix 2 \\
\hline 423 & 0.98920 & 0.44482 & 0.01080 & 0.55518 & 0.00000 & 0.00000 & ethanol/oxygen \\
\hline 433 & 0.98844 & 0.55030 & 0.00000 & 0.00000 & 0.01156 & 0.44970 & ethanol/nitrogen \\
\hline 433 & 0.98790 & 0.53116 & 0.00182 & 0.09177 & 0.01027 & 0.37707 & ethanol/gas mix 1 \\
\hline 433 & 0.98777 & 0.54941 & 0.00663 & 0.33498 & 0.00560 & 0.11561 & ethanol/gas mix 2 \\
\hline 433 & 0.98971 & 0.57413 & 0.01029 & 0.42587 & 0.00000 & 0.00000 & ethanol/oxygen \\
\hline 443 & 0.97950 & 0.66965 & 0.00000 & 0.00000 & 0.02050 & 0.33035 & ethanol/nitrogen \\
\hline 443 & 0.98795 & 0.63795 & 0.00218 & 0.06947 & 0.00987 & 0.29259 & ethanol/gas mix 1 \\
\hline 443 & 0.98183 & 0.65690 & 0.00576 & 0.24991 & 0.01241 & 0.09319 & ethanol/gas mix 2 \\
\hline 443 & 0.99202 & 0.69988 & 0.00798 & 0.30012 & 0.00000 & 0.00000 & ethanol/oxygen \\
\hline 453 & 0.99066 & 0.80752 & 0.00000 & 0.00000 & 0.00934 & 0.19248 & ethanol/nitrogen \\
\hline 453 & 0.98759 & 0.75662 & 0.00211 & 0.04736 & 0.01031 & 0.19602 & ethanol/gas mix 1 \\
\hline 453 & 0.98998 & 0.84709 & 0.01002 & 0.15291 & 0.00000 & 0.00000 & ethanol/oxygen \\
\hline 303 & 0.98204 & 0.02302 & 0.00000 & 0.00000 & 0.01796 & 0.97698 & ethanol/nitrogen \\
\hline 303 & 0.97969 & 0.02602 & 0.00487 & 0.18363 & 0.01543 & 0.79035 & ethanol/gas mix 1 \\
\hline 303 & 0.97071 & 0.00322 & 0.02421 & 0.76946 & 0.00508 & 0.22731 & ethanol/gas mix 2 \\
\hline 303 & 0.97032 & 0.01872 & 0.02968 & 0.98128 & 0.00000 & 0.00000 & ethanol/oxygen \\
\hline 313 & 0.98139 & 0.02640 & 0.00000 & 0.00000 & 0.01861 & 0.97360 & ethanol/nitrogen \\
\hline 313 & 0.98127 & 0.03928 & 0.00340 & 0.18522 & 0.01534 & 0.77550 & ethanol/gas mix 1 \\
\hline 313 & 0.97382 & 0.00063 & 0.02127 & 0.77515 & 0.00491 & 0.22421 & ethanol/gas mix 2 \\
\hline 313 & 0.96983 & 0.01749 & 0.03017 & 0.98251 & 0.00000 & 0.00000 & ethanol/oxygen \\
\hline 323 & 0.98118 & 0.02793 & 0.00000 & 0.00000 & 0.01882 & 0.97207 & ethanol/nitrogen \\
\hline 323 & 0.97940 & 0.04098 & 0.00499 & 0.18214 & 0.01561 & 0.77688 & ethanol/gas mix 1 \\
\hline 323 & 0.97322 & 0.00151 & 0.02181 & 0.77390 & 0.00497 & 0.22459 & ethanol/gas mix 2 \\
\hline 323 & 0.96829 & 0.01477 & 0.03171 & 0.98523 & 0.00000 & 0.00000 & ethanol/oxygen \\
\hline 333 & 0.97790 & 0.02845 & 0.00679 & 0.18930 & 0.01530 & 0.78225 & ethanol/gas mix 1 \\
\hline 333 & 0.97190 & 0.00039 & 0.02335 & 0.78046 & 0.00475 & 0.21915 & ethanol/gas mix 2 \\
\hline 333 & 0.96785 & 0.00008 & 0.03215 & 0.99992 & 0.00000 & 0.00000 & ethanol/oxygen \\
\hline 343 & 0.97672 & 0.03063 & 0.00596 & 0.18409 & 0.01733 & 0.78528 & ethanol/gas mix 1 \\
\hline 343 & 0.97101 & 0.00787 & 0.02401 & 0.76665 & 0.00498 & 0.22548 & ethanol/gas mix 2 \\
\hline 343 & 0.96907 & 0.02520 & 0.03093 & 0.97480 & 0.00000 & 0.00000 & ethanol/oxygen \\
\hline 353 & 0.97727 & 0.04257 & 0.00570 & 0.17976 & 0.01703 & 0.77766 & ethanol/gas mix 1 \\
\hline 353 & 0.97108 & 0.02166 & 0.02367 & 0.75630 & 0.00525 & 0.22204 & ethanol/gas mix 2 \\
\hline 353 & 0.96961 & 0.03471 & 0.03039 & 0.96529 & 0.00000 & 0.00000 & ethanol/oxygen \\
\hline 363 & 0.97957 & 0.05503 & 0.00333 & 0.17918 & 0.01710 & 0.76579 & ethanol/gas mix 1 \\
\hline 363 & 0.97246 & 0.03347 & 0.02199 & 0.75773 & 0.00555 & 0.20881 & ethanol/gas mix 2 \\
\hline 363 & 0.97167 & 0.06825 & 0.02833 & 0.93175 & 0.00000 & 0.00000 & ethanol/oxygen \\
\hline 373 & 0.97872 & 0.07575 & 0.00384 & 0.17444 & 0.01745 & 0.74981 & ethanol/gas mix 1 \\
\hline 373 & 0.97232 & 0.05567 & 0.02248 & 0.73054 & 0.00521 & 0.21379 & ethanol/gas mix 2 \\
\hline 373 & 0.96932 & 0.08114 & 0.03068 & 0.91886 & 0.00000 & 0.00000 & ethano/oxygen \\
\hline 383 & 0.97858 & 0.10589 & 0.00341 & 0.17230 & 0.01802 & 0.72181 & ethanol/gas mix 1 \\
\hline 383 & 0.97254 & 0.09611 & 0.02180 & 0.69841 & 0.00566 & 0.20548 & ethanol/gas mix 2 \\
\hline 383 & 0.97086 & 0.08386 & 0.02914 & 0.91614 & 0.00000 & 0.00000 & ethanol/oxygen \\
\hline 393 & 0.97705 & 0.11428 & 0.00370 & 0.16739 & 0.01925 & 0.71833 & ethanol/gas mix 1 \\
\hline 393 & 0.97134 & 0.11605 & 0.02377 & 0.68219 & 0.00488 & 0.20176 & ethanol/gas mix 2 \\
\hline 393 & 0.96903 & 0.13429 & 0.03097 & 0.86571 & 0.00000 & 0.00000 & ethanol/oxygen \\
\hline 403 & 0.97713 & 0.16307 & 0.00331 & 0.16102 & 0.01957 & 0.67591 & ethanol/gas mix 1 \\
\hline 403 & 0.97188 & 0.15852 & 0.02196 & 0.65079 & 0.00615 & 0.19069 & ethanol/gas mix 2 \\
\hline 403 & 0.97023 & 0.17012 & 0.02977 & 0.82988 & 0.00000 & 0.00000 & ethanol/oxygen \\
\hline 413 & 0.97657 & 0.22806 & 0.00415 & 0.14977 & 0.01928 & 0.62217 & ethanol/gas mix 1 \\
\hline 413 & 0.97201 & 0.22256 & 0.02233 & 0.60673 & 0.00566 & 0.17070 & ethanol/gas mix 2 \\
\hline 413 & 0.97133 & 0.25146 & 0.02867 & 0.74854 & 0.00000 & 0.00000 & ethanol/oxygen \\
\hline 423 & 0.97669 & 0.30057 & 0.00000 & 0.00000 & 0.02331 & 0.69943 & ethanol/nitrogen \\
\hline 423 & 0.97621 & 0.28888 & 0.00403 & 0.13915 & 0.01976 & 0.57196 & ethanol/gas mix 1 \\
\hline
\end{tabular}




\begin{tabular}{|c|c|c|c|c|c|c|c|}
\hline 423 & 0.97252 & 0.27463 & 0.02179 & 0.56030 & 0.00569 & 0.16507 & ethanol/gas mix 2 \\
\hline 423 & 0.96977 & 0.31731 & 0.03023 & 0.68269 & 0.00000 & 0.00000 & ethanol/oxygen \\
\hline 433 & 0.97627 & 0.38524 & 0.00000 & 0.00000 & 0.02373 & 0.61476 & ethanol/nitrogen \\
\hline 433 & 0.97712 & 0.37224 & 0.00431 & 0.12555 & 0.01857 & 0.50221 & ethanol/gas mix 1 \\
\hline 433 & 0.97480 & 0.34370 & 0.02039 & 0.51141 & 0.00481 & 0.14489 & ethanol/gas mix 2 \\
\hline 433 & 0.97134 & 0.37585 & 0.02866 & 0.62415 & 0.00000 & 0.00000 & ethanol/oxygen \\
\hline 443 & 0.97673 & 0.46728 & 0.00000 & 0.00000 & 0.02327 & 0.53272 & ethanol/nitrogen \\
\hline 443 & 0.97764 & 0.45485 & 0.00220 & 0.11068 & 0.02015 & 0.43447 & ethanol/gas mix 1 \\
\hline 443 & 0.97391 & 0.42370 & 0.02111 & 0.44210 & 0.00498 & 0.13420 & ethanol/gas mix 2 \\
\hline 443 & 0.97146 & 0.47582 & 0.02854 & 0.52418 & 0.00000 & 0.00000 & ethanol/oxygen \\
\hline 453 & 0.97323 & 0.56079 & 0.00000 & 0.00000 & 0.02677 & 0.43921 & ethanol/nitrogen \\
\hline 453 & 0.97775 & 0.54911 & 0.00365 & 0.09169 & 0.01861 & 0.35919 & ethanol/gas mix 1 \\
\hline 453 & 0.97041 & 0.49529 & 0.02246 & 0.40326 & 0.00713 & 0.10145 & ethanol/gas mix 2 \\
\hline 453 & 0.97458 & 0.56023 & 0.02542 & 0.43977 & 0.00000 & 0.00000 & ethanol/oxygen \\
\hline 463 & 0.97867 & 0.67169 & 0.00000 & 0.00000 & 0.02133 & 0.32831 & ethanol/nitrogen \\
\hline 463 & 0.98138 & 0.63897 & 0.00154 & 0.07530 & 0.01708 & 0.28573 & ethanol/gas mix 1 \\
\hline 463 & 0.97409 & 0.61710 & 0.01895 & 0.29227 & 0.00696 & 0.09063 & ethanol/gas mix 2 \\
\hline 463 & 0.97544 & 0.65298 & 0.02456 & 0.34702 & 0.00000 & 0.00000 & ethanol/oxygen \\
\hline 473 & 0.98278 & 0.76326 & 0.00000 & 0.00000 & 0.01722 & 0.23674 & ethanol/nitrogen \\
\hline 473 & 0.98194 & 0.74766 & 0.00276 & 0.05028 & 0.01530 & 0.20206 & ethanol/gas mix 1 \\
\hline 303 & 0.97524 & 0.00613 & 0.00569 & 0.18492 & 0.01906 & 0.80895 & ethanol/gas mix 1 \\
\hline 303 & 0.96800 & 0.00071 & 0.02580 & 0.76905 & 0.00620 & 0.23023 & ethanol/gas mix 2 \\
\hline 303 & 0.96385 & 0.01735 & 0.03615 & 0.98265 & 0.00000 & 0.00000 & ethanol/oxygen \\
\hline 313 & 0.97609 & 0.01227 & 0.00568 & 0.18123 & 0.01823 & 0.80651 & ethanol/gas mix 1 \\
\hline 313 & 0.96664 & 0.00269 & 0.02717 & 0.77043 & 0.00619 & 0.22688 & ethanol/gas mix 2 \\
\hline 313 & 0.96550 & 0.00106 & 0.03450 & 0.99894 & 0.00000 & 0.00000 & ethanol/oxygen \\
\hline 323 & 0.97452 & 0.01097 & 0.00640 & 0.18218 & 0.01908 & 0.80684 & ethanol/gas mix 1 \\
\hline 323 & 0.96614 & 0.00417 & 0.02818 & 0.76878 & 0.00567 & 0.22705 & ethanol/gas mix 2 \\
\hline 323 & 0.96370 & 0.01126 & 0.03630 & 0.98874 & 0.00000 & 0.00000 & ethanol/oxygen \\
\hline 333 & 0.97365 & 0.00369 & 0.00718 & 0.18454 & 0.01918 & 0.81177 & ethanol/gas mix 1 \\
\hline 333 & 0.96452 & 0.00594 & 0.02954 & 0.76700 & 0.00595 & 0.22707 & ethanol/gas mix 2 \\
\hline 333 & 0.96303 & 0.01264 & 0.03697 & 0.98736 & 0.00000 & 0.00000 & ethanol/oxygen \\
\hline 343 & 0.97365 & 0.02408 & 0.00675 & 0.18352 & 0.01960 & 0.79239 & ethanol/gas mix 1 \\
\hline 343 & 0.96506 & 0.01732 & 0.02904 & 0.75882 & 0.00589 & 0.22386 & ethanol/gas mix 2 \\
\hline 343 & 0.96357 & 0.03264 & 0.03643 & 0.96736 & 0.00000 & 0.00000 & ethanol/oxygen \\
\hline 353 & 0.97211 & 0.01516 & 0.00770 & 0.18203 & 0.02019 & 0.80281 & ethanol/gas mix 1 \\
\hline 353 & 0.96563 & 0.02847 & 0.02839 & 0.74779 & 0.00598 & 0.22374 & ethanol/gas mix 2 \\
\hline 353 & 0.96290 & 0.00186 & 0.03710 & 0.99814 & 0.00000 & 0.00000 & ethanol/oxygen \\
\hline 363 & 0.97462 & 0.04663 & 0.00454 & 0.17749 & 0.02085 & 0.77588 & ethanol/gas mix 1 \\
\hline 363 & 0.96765 & 0.04441 & 0.02674 & 0.73364 & 0.00560 & 0.22195 & ethanol/gas mix 2 \\
\hline 363 & 0.96409 & 0.06515 & 0.03591 & 0.93485 & 0.00000 & 0.00000 & ethanol/oxygen \\
\hline 373 & 0.97292 & 0.03692 & 0.00610 & 0.18650 & 0.02098 & 0.77658 & ethanol/gas mix 1 \\
\hline 373 & 0.96570 & 0.05990 & 0.02769 & 0.72592 & 0.00662 & 0.21418 & ethanol/gas mix 2 \\
\hline 373 & 0.96467 & 0.06714 & 0.03533 & 0.93286 & 0.00000 & 0.00000 & ethanol/oxygen \\
\hline 383 & 0.97361 & 0.08547 & 0.00441 & 0.17702 & 0.02198 & 0.73751 & ethanol/gas mix 1 \\
\hline 383 & 0.96679 & 0.07061 & 0.02619 & 0.71746 & 0.00702 & 0.21193 & ethanol/gas mix 2 \\
\hline 383 & 0.96464 & 0.07009 & 0.03536 & 0.92991 & 0.00000 & 0.00000 & ethanol/oxygen \\
\hline 393 & 0.97201 & 0.12188 & 0.00527 & 0.17195 & 0.02272 & 0.70616 & ethanol/gas mix 1 \\
\hline 393 & 0.96589 & 0.11641 & 0.02830 & 0.67957 & 0.00582 & 0.20402 & ethanol/gas mix 2 \\
\hline 393 & 0.96361 & 0.09583 & 0.03639 & 0.90417 & 0.00000 & 0.00000 & ethanol/oxygen \\
\hline 403 & 0.97244 & 0.14623 & 0.00390 & 0.16652 & 0.02366 & 0.68725 & ethanol/gas mix 1 \\
\hline 403 & 0.96492 & 0.15992 & 0.02858 & 0.64636 & 0.00650 & 0.19371 & ethanol/gas mix 2 \\
\hline 403 & 0.96453 & 0.16538 & 0.03547 & 0.83462 & 0.00000 & 0.00000 & ethano//oxygen \\
\hline 413 & 0.96880 & 0.19891 & 0.00558 & 0.15297 & 0.02562 & 0.64812 & ethanol/gas mix 1 \\
\hline 413 & 0.96545 & 0.19817 & 0.02789 & 0.62122 & 0.00666 & 0.18062 & ethanol/gas mix 2 \\
\hline 413 & 0.96414 & 0.19910 & 0.03586 & 0.80090 & 0.00000 & 0.00000 & ethanol/oxygen \\
\hline 423 & 0.97075 & 0.24485 & 0.00479 & 0.14801 & 0.02445 & 0.60714 & ethanol/gas mix 1 \\
\hline 423 & 0.96673 & 0.24909 & 0.02653 & 0.58256 & 0.00674 & 0.16836 & ethanol/gas mix 2 \\
\hline 423 & 0.96356 & 0.28433 & 0.03644 & 0.71567 & 0.00000 & 0.00000 & ethanol/oxygen \\
\hline 433 & 0.96901 & 0.31838 & 0.00512 & 0.13611 & 0.02587 & 0.54551 & ethanol/gas mix 1 \\
\hline 433 & 0.96471 & 0.31087 & 0.02845 & 0.52587 & 0.00683 & 0.16326 & ethanol/gas mix 2 \\
\hline 433 & 0.96423 & 0.35153 & 0.03577 & 0.64847 & 0.00000 & 0.00000 & ethanol/oxygen \\
\hline 443 & 0.96871 & 0.39601 & 0.00423 & 0.11912 & 0.02706 & 0.48487 & ethanol/gas mix 1 \\
\hline 443 & 0.96678 & 0.38085 & 0.02508 & 0.49992 & 0.00815 & 0.11923 & ethanol/gas mix 2 \\
\hline 443 & 0.96476 & 0.43103 & 0.03524 & 0.56897 & 0.00000 & 0.00000 & ethanol/oxygen \\
\hline 453 & 0.96945 & 0.48629 & 0.00456 & 0.09995 & 0.02599 & 0.41376 & ethanol/gas mix 1 \\
\hline 453 & 0.96544 & 0.45796 & 0.02664 & 0.40192 & 0.00792 & 0.14012 & ethanol/gas mix 2 \\
\hline 453 & 0.96651 & 0.49923 & 0.03349 & 0.50077 & 0.00000 & 0.00000 & ethanol/oxygen \\
\hline 463 & 0.96883 & 0.56673 & 0.00447 & 0.08900 & 0.02670 & 0.34427 & ethanol/gas mix 1 \\
\hline 463 & 0.96709 & 0.54324 & 0.02538 & 0.33789 & 0.00753 & 0.11886 & ethanol/gas mix 2 \\
\hline 463 & 0.96706 & 0.60293 & 0.03294 & 0.39707 & 0.00000 & 0.00000 & ethanol/oxygen \\
\hline 473 & 0.97241 & 0.66592 & 0.00434 & 0.06595 & 0.02325 & 0.26813 & ethanol/gas mix 1 \\
\hline 473 & 0.97025 & 0.65133 & 0.02294 & 0.25565 & 0.00680 & 0.09302 & ethanol/gas mix 2 \\
\hline 473 & 0.96931 & 0.70215 & 0.03069 & 0.29785 & 0.00000 & 0.00000 & ethanol/oxygen \\
\hline 303 & 0.98064 & 0.01429 & 0.00000 & 0.00000 & 0.01936 & 0.98571 & ethanol/nitrogen \\
\hline 303 & 0.97452 & 0.00790 & 0.00548 & 0.18711 & 0.02000 & 0.80499 & ethanol/gas mix 1 \\
\hline 303 & 0.96490 & 0.00721 & 0.02812 & 0.74478 & 0.00698 & 0.24801 & ethanol/gas mix 2 \\
\hline 303 & 0.96148 & 0.00961 & 0.03852 & 0.99039 & 0.00000 & 0.00000 & ethanol/oxygen \\
\hline 313 & 0.97977 & 0.01548 & 0.00000 & 0.00000 & 0.02023 & 0.98452 & ethanol/nitrogen \\
\hline
\end{tabular}




\begin{tabular}{|c|c|c|c|c|c|c|c|c|}
\hline 6.5 & 313 & 0.97525 & 0.01233 & 0.00463 & 0.19070 & 0.02012 & 0.79697 & ethanol/gas mix 1 \\
\hline 6.5 & 313 & 0.96594 & 0.01748 & 0.02728 & 0.74031 & 0.00678 & 0.24222 & ethanol/gas mix 2 \\
\hline 6.5 & 313 & 0.96212 & 0.00560 & 0.03788 & 0.99440 & 0.00000 & 0.00000 & ethanol/oxygen \\
\hline 6.5 & 323 & 0.97870 & 0.02159 & 0.00000 & 0.00000 & 0.02130 & 0.97841 & ethanol/nitrogen \\
\hline 6.5 & 323 & 0.97367 & 0.02199 & 0.00524 & 0.18697 & 0.02109 & 0.79104 & ethanol/gas mix 1 \\
\hline 6.5 & 323 & 0.96333 & 0.00690 & 0.02979 & 0.76543 & 0.00688 & 0.22767 & ethanol/gas mix 2 \\
\hline 6.5 & 323 & 0.96180 & 0.00576 & 0.03820 & 0.99424 & 0.00000 & 0.00000 & ethanol/oxygen \\
\hline 6.5 & 333 & 0.97818 & 0.02904 & 0.00000 & 0.00000 & 0.02182 & 0.97096 & ethanol/nitrogen \\
\hline 6.5 & 333 & 0.97192 & 0.01870 & 0.00658 & 0.18438 & 0.02150 & 0.79691 & ethanol/gas mix 1 \\
\hline 6.5 & 333 & 0.96200 & 0.00314 & 0.03147 & 0.76636 & 0.00653 & 0.23050 & ethanol/gas mix 2 \\
\hline 6.5 & 333 & 0.96062 & 0.00448 & 0.03938 & 0.99552 & 0.00000 & 0.00000 & ethanol/oxygen \\
\hline 6.5 & 343 & 0.97813 & 0.03397 & 0.00000 & 0.00000 & 0.02187 & 0.96603 & ethanol/nitrogen \\
\hline 6.5 & 343 & 0.97165 & 0.01761 & 0.00724 & 0.18788 & 0.02111 & 0.79451 & ethanol/gas mix 1 \\
\hline 6.5 & 343 & 0.96353 & 0.00384 & 0.02996 & 0.76567 & 0.00651 & 0.23048 & ethanol/gas mix 2 \\
\hline 6.5 & 343 & 0.96109 & 0.03048 & 0.03891 & 0.96952 & 0.00000 & 0.00000 & ethanol/oxygen \\
\hline 6.5 & 353 & 0.97736 & 0.04806 & 0.00000 & 0.00000 & 0.02264 & 0.95194 & ethanol/nitrogen \\
\hline 6.5 & 353 & 0.96973 & 0.02494 & 0.00791 & 0.18508 & 0.02236 & 0.78998 & ethanol/gas mix 1 \\
\hline 6.5 & 353 & 0.96231 & 0.02426 & 0.03097 & 0.74830 & 0.00672 & 0.22744 & ethanol/gas mix 2 \\
\hline 6.5 & 353 & 0.96010 & 0.04521 & 0.03990 & 0.95479 & 0.00000 & 0.00000 & ethanol/oxygen \\
\hline 6.5 & 363 & 0.97716 & 0.05429 & 0.00000 & 0.00000 & 0.02284 & 0.94571 & ethanol/nitrogen \\
\hline 6.5 & 363 & 0.97218 & 0.04107 & 0.00408 & 0.18328 & 0.02374 & 0.77565 & ethanol/gas mix 1 \\
\hline 6.5 & 363 & 0.96367 & 0.03410 & 0.02915 & 0.72941 & 0.00718 & 0.23649 & ethanol/gas mix 2 \\
\hline 6.5 & 363 & 0.96262 & 0.05439 & 0.03738 & 0.94561 & 0.00000 & 0.00000 & ethanol/oxygen \\
\hline 6.5 & 373 & 0.97575 & 0.07708 & 0.00000 & 0.00000 & 0.02425 & 0.92292 & ethanol/nitrogen \\
\hline 6.5 & 373 & 0.97120 & 0.06039 & 0.00469 & 0.17907 & 0.02412 & 0.76054 & ethanol/gas mix 1 \\
\hline 6.5 & 373 & 0.96259 & 0.05877 & 0.03029 & 0.71034 & 0.00712 & 0.23089 & ethanol/gas mix 2 \\
\hline 6.5 & 373 & 0.96219 & 0.06669 & 0.03781 & 0.93331 & 0.00000 & 0.00000 & ethanol/oxygen \\
\hline 6.5 & 383 & 0.97498 & 0.09461 & 0.00000 & 0.00000 & 0.02502 & 0.90539 & ethanol/nitrogen \\
\hline 6.5 & 383 & 0.97158 & 0.08339 & 0.00386 & 0.17467 & 0.02456 & 0.74194 & ethanol/gas mix 1 \\
\hline 6.5 & 383 & 0.96098 & 0.05993 & 0.03108 & 0.72624 & 0.00794 & 0.21383 & ethanol/gas mix 2 \\
\hline 6.5 & 383 & 0.96191 & 0.08807 & 0.03809 & 0.91193 & 0.00000 & 0.00000 & ethanol/oxygen \\
\hline 6.5 & 393 & 0.97488 & 0.12380 & 0.00000 & 0.00000 & 0.02512 & 0.87620 & ethanol/nitrogen \\
\hline 6.5 & 393 & 0.96965 & 0.10963 & 0.00533 & 0.17150 & 0.02502 & 0.71887 & ethanol/gas mix 1 \\
\hline 6.5 & 393 & 0.96154 & 0.10825 & 0.03108 & 0.67742 & 0.00738 & 0.21433 & ethanol/gas mix 2 \\
\hline 6.5 & 393 & 0.96092 & 0.13083 & 0.03908 & 0.86917 & 0.00000 & 0.00000 & ethanol/oxygen \\
\hline 6.5 & 403 & 0.97358 & 0.15367 & 0.00000 & 0.00000 & 0.02642 & 0.84633 & ethanol/nitrogen \\
\hline 6.5 & 403 & 0.96947 & 0.13963 & 0.00473 & 0.16324 & 0.02581 & 0.69713 & ethanol/gas mix 1 \\
\hline 6.5 & 403 & 0.96277 & 0.13201 & 0.02992 & 0.65478 & 0.00731 & 0.21321 & ethanol/gas mix 2 \\
\hline 6.5 & 403 & 0.95839 & 0.14475 & 0.04161 & 0.85525 & 0.00000 & 0.00000 & ethanol/oxygen \\
\hline 6.5 & 413 & 0.97311 & 0.19941 & 0.00000 & 0.00000 & 0.02689 & 0.80059 & ethanol/nitrogen \\
\hline 6.5 & 413 & 0.96813 & 0.18612 & 0.00541 & 0.15609 & 0.02646 & 0.65779 & ethanol/gas mix 1 \\
\hline 6.5 & 413 & 0.96256 & 0.18580 & 0.03067 & 0.61913 & 0.00678 & 0.19507 & ethanol/gas mix 2 \\
\hline 6.5 & 413 & 0.95805 & 0.19675 & 0.04195 & 0.80325 & 0.00000 & 0.00000 & ethanol/oxygen \\
\hline 6.5 & 423 & 0.97209 & 0.25594 & 0.00000 & 0.00000 & 0.02791 & 0.74406 & ethanol/nitrogen \\
\hline 6.5 & 423 & 0.96719 & 0.24313 & 0.00513 & 0.14604 & 0.02768 & 0.61083 & ethanol/gas mix 1 \\
\hline 6.5 & 423 & 0.96006 & 0.22660 & 0.03168 & 0.57461 & 0.00826 & 0.19879 & ethanol/gas mix 2 \\
\hline 6.5 & 423 & 0.96059 & 0.24606 & 0.03941 & 0.75394 & 0.00000 & 0.00000 & ethanol/oxygen \\
\hline 6.5 & 433 & 0.97193 & 0.31734 & 0.00000 & 0.00000 & 0.02807 & 0.68266 & ethanol/nitrogen \\
\hline 6.5 & 433 & 0.96620 & 0.30497 & 0.00515 & 0.13191 & 0.02865 & 0.56312 & ethanol/gas mix 1 \\
\hline 6.5 & 433 & 0.95870 & 0.30719 & 0.03250 & 0.53810 & 0.00880 & 0.15470 & ethanol/gas mix 2 \\
\hline 6.5 & 433 & 0.95951 & 0.29160 & 0.04049 & 0.70840 & 0.00000 & 0.00000 & ethanol/oxygen \\
\hline 6.5 & 443 & 0.97190 & 0.38137 & 0.00000 & 0.00000 & 0.02810 & 0.61863 & ethanol/nitrogen \\
\hline 6.5 & 443 & 0.96501 & 0.37028 & 0.00488 & 0.12407 & 0.03010 & 0.50565 & ethanol/gas mix 1 \\
\hline 6.5 & 443 & 0.95741 & 0.31670 & 0.03252 & 0.52181 & 0.01007 & 0.16149 & ethanol/gas mix 2 \\
\hline 6.5 & 443 & 0.95879 & 0.36204 & 0.04121 & 0.63796 & 0.00000 & 0.00000 & ethanol/oxygen \\
\hline 6.5 & 453 & 0.97158 & 0.46514 & 0.00000 & 0.00000 & 0.02842 & 0.53486 & ethanol/nitrogen \\
\hline 6.5 & 453 & 0.96184 & 0.45487 & 0.00645 & 0.10477 & 0.03171 & 0.44036 & ethanol/gas mix 1 \\
\hline 6.5 & 453 & 0.95969 & 0.45578 & 0.03174 & 0.41630 & 0.00858 & 0.12791 & ethanol/gas mix 2 \\
\hline 6.5 & 453 & 0.95836 & 0.45564 & 0.04164 & 0.54436 & 0.00000 & 0.00000 & ethanol/oxygen \\
\hline 6.5 & 463 & 0.97073 & 0.55953 & 0.00000 & 0.00000 & 0.02927 & 0.44047 & ethanol/nitrogen \\
\hline 6.5 & 463 & 0.96880 & 0.52715 & 0.00303 & 0.09118 & 0.02817 & 0.38167 & ethanol/gas mix 1 \\
\hline 6.5 & 463 & 0.96151 & 0.52896 & 0.02963 & 0.35749 & 0.00886 & 0.11355 & ethanol/gas mix 2 \\
\hline 6.5 & 463 & 0.95699 & 0.54422 & 0.04301 & 0.45578 & 0.00000 & 0.00000 & ethanol/oxygen \\
\hline 6.5 & 473 & 0.97563 & 0.66346 & 0.00000 & 0.00000 & 0.02437 & 0.33654 & ethanol/nitrogen \\
\hline 6.5 & 473 & 0.96764 & 0.62868 & 0.00483 & 0.07380 & 0.02753 & 0.29752 & ethanol/gas mix 1 \\
\hline 6.5 & 473 & 0.96371 & 0.60993 & 0.02411 & 0.29397 & 0.01218 & 0.09611 & ethanol/gas mix 2 \\
\hline 6.5 & 473 & 0.96259 & 0.62858 & 0.03741 & 0.37142 & 0.00000 & 0.00000 & ethano/oxygen \\
\hline 8 & 303 & 0.97355 & 0.01106 & 0.00000 & 0.00000 & 0.02645 & 0.98894 & ethanol/nitrogen \\
\hline 8 & 303 & 0.96904 & 0.00328 & 0.00743 & 0.18980 & 0.02353 & 0.80692 & ethanol/gas mix 1 \\
\hline 8 & 303 & 0.95737 & 0.00876 & 0.03498 & 0.76017 & 0.00765 & 0.23107 & ethanol/gas mix 2 \\
\hline 8 & 303 & 0.95371 & 0.00825 & 0.04629 & 0.99175 & 0.00000 & 0.00000 & ethanol/oxygen \\
\hline 8 & 313 & 0.97391 & 0.00634 & 0.00000 & 0.00000 & 0.02609 & 0.99366 & ethanol/nitrogen \\
\hline 8 & 313 & 0.96857 & 0.01042 & 0.00713 & 0.18807 & 0.02431 & 0.80151 & ethanol/gas mix 1 \\
\hline 8 & 313 & 0.95625 & 0.00935 & 0.03598 & 0.75358 & 0.00777 & 0.23708 & ethanol/gas mix 2 \\
\hline 8 & 313 & 0.95395 & 0.00512 & 0.04605 & 0.99488 & 0.00000 & 0.00000 & ethanol/oxygen \\
\hline 8 & 323 & 0.97318 & 0.00905 & 0.00000 & 0.00000 & 0.02682 & 0.99095 & ethanol/nitrogen \\
\hline 8 & 323 & 0.96682 & 0.01868 & 0.00811 & 0.18515 & 0.02506 & 0.79617 & ethanol/gas mix 1 \\
\hline 8 & 323 & 0.95766 & 0.01193 & 0.03478 & 0.75473 & 0.00756 & 0.23334 & ethanol/gas mix 2 \\
\hline 8 & 323 & 0.95120 & 0.00789 & 0.04880 & 0.99211 & 0.00000 & 0.00000 & ethanol/oxygen \\
\hline
\end{tabular}




\begin{tabular}{|c|c|c|c|c|c|c|c|c|}
\hline 8 & 333 & 0.97218 & 0.00660 & 0.00000 & 0.00000 & 0.02782 & 0.99340 & ethanol/nitrogen \\
\hline 8 & 333 & 0.96555 & 0.01337 & 0.00935 & 0.18249 & 0.02510 & 0.80414 & ethanol/gas mix 1 \\
\hline 8 & 333 & 0.95515 & 0.00198 & 0.03736 & 0.76638 & 0.00749 & 0.23164 & ethanol/gas mix 2 \\
\hline 8 & 333 & 0.95059 & 0.00777 & 0.04941 & 0.99223 & 0.00000 & 0.00000 & ethanol/oxygen \\
\hline 8 & 343 & 0.97162 & 0.01129 & 0.00000 & 0.00000 & 0.02838 & 0.98871 & ethanol/nitrogen \\
\hline 8 & 343 & 0.96452 & 0.02348 & 0.00910 & 0.18787 & 0.02638 & 0.78866 & ethanol/gas mix 1 \\
\hline 8 & 343 & 0.95594 & 0.00908 & 0.03616 & 0.76343 & 0.00791 & 0.22749 & ethanol/gas mix 2 \\
\hline 8 & 343 & 0.95078 & 0.00272 & 0.04922 & 0.99728 & 0.00000 & 0.00000 & ethanol/oxygen \\
\hline 8 & 353 & 0.97069 & 0.02802 & 0.00000 & 0.00000 & 0.02931 & 0.97198 & ethanol/nitrogen \\
\hline 8 & 353 & 0.96341 & 0.03229 & 0.00970 & 0.18575 & 0.02689 & 0.78196 & ethanol/gas mix 1 \\
\hline 8 & 353 & 0.95455 & 0.00999 & 0.03637 & 0.76116 & 0.00908 & 0.22885 & ethanol/gas mix 2 \\
\hline 8 & 353 & 0.95074 & 0.00022 & 0.04926 & 0.99978 & 0.00000 & 0.00000 & ethanol/oxygen \\
\hline 8 & 363 & 0.96987 & 0.03839 & 0.00000 & 0.00000 & 0.03013 & 0.96161 & ethanol/nitrogen \\
\hline 8 & 363 & 0.96437 & 0.04159 & 0.00751 & 0.18434 & 0.02812 & 0.77407 & ethanol/gas mix 1 \\
\hline 8 & 363 & 0.95520 & 0.00892 & 0.03596 & 0.75795 & 0.00884 & 0.23313 & ethanol/gas mix 2 \\
\hline 8 & 363 & 0.95222 & 0.00142 & 0.04778 & 0.99858 & 0.00000 & 0.00000 & ethanol/oxygen \\
\hline 8 & 373 & 0.96904 & 0.06804 & 0.00000 & 0.00000 & 0.03096 & 0.93196 & ethanol/nitrogen \\
\hline 8 & 373 & 0.96370 & 0.05420 & 0.00751 & 0.18442 & 0.02879 & 0.76138 & ethanol/gas mix 1 \\
\hline 8 & 373 & 0.95362 & 0.04842 & 0.03727 & 0.74510 & 0.00912 & 0.20648 & ethanol/gas mix 2 \\
\hline 8 & 373 & 0.95159 & 0.03257 & 0.04841 & 0.96743 & 0.00000 & 0.00000 & ethano//oxygen \\
\hline 8 & 383 & 0.96718 & 0.06379 & 0.00000 & 0.00000 & 0.03282 & 0.93621 & ethanol/nitrogen \\
\hline 8 & 383 & 0.96358 & 0.06905 & 0.00658 & 0.18041 & 0.02983 & 0.75053 & ethanol/gas mix 1 \\
\hline 8 & 383 & 0.95504 & 0.03978 & 0.03672 & 0.74829 & 0.00824 & 0.21193 & ethanol/gas mix 2 \\
\hline 8 & 383 & 0.95011 & 0.06430 & 0.04989 & 0.93570 & 0.00000 & 0.00000 & ethanol/oxygen \\
\hline 8 & 393 & 0.96600 & 0.11057 & 0.00000 & 0.00000 & 0.03400 & 0.88943 & ethanol/nitrogen \\
\hline 8 & 393 & 0.96148 & 0.10244 & 0.00808 & 0.17328 & 0.03044 & 0.72428 & ethanol/gas mix 1 \\
\hline 8 & 393 & 0.95219 & 0.05983 & 0.03874 & 0.72765 & 0.00907 & 0.21252 & ethanol/gas mix 2 \\
\hline 8 & 393 & 0.94805 & 0.09806 & 0.05195 & 0.90194 & 0.00000 & 0.00000 & ethanol/oxygen \\
\hline 8 & 403 & 0.96532 & 0.13375 & 0.00000 & 0.00000 & 0.03468 & 0.86625 & ethanol/nitrogen \\
\hline 8 & 403 & 0.96095 & 0.13312 & 0.00699 & 0.16633 & 0.03205 & 0.70055 & ethanol/gas mix 1 \\
\hline 8 & 403 & 0.95184 & 0.14104 & 0.03821 & 0.66395 & 0.00995 & 0.19501 & ethanol/gas mix 2 \\
\hline 8 & 403 & 0.94916 & 0.12122 & 0.05084 & 0.87878 & 0.00000 & 0.00000 & ethanol/oxygen \\
\hline 8 & 413 & 0.96435 & 0.16906 & 0.00000 & 0.00000 & 0.03565 & 0.83094 & ethanol/nitrogen \\
\hline 8 & 413 & 0.96026 & 0.17174 & 0.00741 & 0.16388 & 0.03233 & 0.66438 & ethanol/gas mix 1 \\
\hline 8 & 413 & 0.95035 & 0.15369 & 0.03944 & 0.65740 & 0.01021 & 0.18890 & ethanol/gas mix 2 \\
\hline 8 & 413 & 0.94872 & 0.14198 & 0.05128 & 0.85802 & 0.00000 & 0.00000 & ethanol/oxygen \\
\hline 8 & 423 & 0.96316 & 0.20441 & 0.00000 & 0.00000 & 0.03684 & 0.79559 & ethanol/nitrogen \\
\hline 8 & 423 & 0.95821 & 0.22187 & 0.00783 & 0.15377 & 0.03396 & 0.62436 & ethanol/gas mix 1 \\
\hline 8 & 423 & 0.94776 & 0.19565 & 0.04225 & 0.61938 & 0.00999 & 0.18497 & ethanol/gas mix 2 \\
\hline 8 & 423 & 0.94836 & 0.15932 & 0.05164 & 0.84068 & 0.00000 & 0.00000 & ethanol/oxygen \\
\hline 8 & 433 & 0.96145 & 0.26763 & 0.00000 & 0.00000 & 0.03855 & 0.73237 & ethanol/nitrogen \\
\hline 8 & 433 & 0.95702 & 0.27434 & 0.00778 & 0.14301 & 0.03520 & 0.58266 & ethanol/gas mix 1 \\
\hline 8 & 433 & 0.94870 & 0.26412 & 0.04124 & 0.55803 & 0.01006 & 0.17786 & ethanol/gas mix 2 \\
\hline 8 & 433 & 0.94809 & 0.18230 & 0.05191 & 0.81770 & 0.00000 & 0.00000 & ethanol/oxygen \\
\hline 8 & 443 & 0.96071 & 0.33449 & 0.00000 & 0.00000 & 0.03929 & 0.66551 & ethanol/nitrogen \\
\hline 8 & 443 & 0.95474 & 0.33465 & 0.00822 & 0.13153 & 0.03704 & 0.53382 & ethanol/gas mix 1 \\
\hline 8 & 443 & 0.94837 & 0.28871 & 0.04138 & 0.55434 & 0.01025 & 0.15695 & ethanol/gas mix 2 \\
\hline 8 & 443 & 0.94664 & 0.22793 & 0.05336 & 0.77207 & 0.00000 & 0.00000 & ethanol/oxygen \\
\hline 8 & 453 & 0.96043 & 0.40776 & 0.00000 & 0.00000 & 0.03957 & 0.59224 & ethanol/nitrogen \\
\hline 8 & 453 & 0.95395 & 0.39950 & 0.00880 & 0.12118 & 0.03725 & 0.47932 & ethanol/gas mix 1 \\
\hline 8 & 453 & 0.94407 & 0.36423 & 0.04511 & 0.48998 & 0.01082 & 0.14579 & ethanol/gas mix 2 \\
\hline 8 & 453 & 0.94597 & 0.33240 & 0.05403 & 0.66760 & 0.00000 & 0.00000 & ethanol/oxygen \\
\hline 8 & 463 & 0.95934 & 0.49059 & 0.00000 & 0.00000 & 0.04066 & 0.50941 & ethanol/nitrogen \\
\hline 8 & 463 & 0.95410 & 0.48569 & 0.00783 & 0.10351 & 0.03807 & 0.41081 & ethanol/gas mix 1 \\
\hline 8 & 463 & 0.93891 & 0.44440 & 0.04856 & 0.42981 & 0.01254 & 0.12579 & ethanol/gas mix 2 \\
\hline 8 & 463 & 0.94480 & 0.46537 & 0.05520 & 0.53463 & 0.00000 & 0.00000 & ethanol/oxygen \\
\hline 8 & 473 & 0.96115 & 0.58115 & 0.00000 & 0.00000 & 0.03885 & 0.41885 & ethanol/nitrogen \\
\hline 8 & 473 & 0.95394 & 0.55157 & 0.00838 & 0.08688 & 0.03768 & 0.36155 & ethanol/gas mix 1 \\
\hline 8 & 473 & 0.94416 & 0.52696 & 0.04403 & 0.36993 & 0.01181 & 0.10311 & ethanol/gas mix 2 \\
\hline 8 & 473 & 0.94320 & 0.55807 & 0.05680 & 0.44193 & 0.00000 & 0.00000 & ethanol/oxygen \\
\hline
\end{tabular}




\section{VIII - List of symbols}

$a$ : phase-dependent PR-EOS mixture parameter

$a_{\mathrm{i}-\mathrm{i}} / a_{\mathrm{i}-\mathrm{j}}$ : phase-independent PR-EOS pure/mixture parameter

$b$ : phase-dependent PR-EOS mixture parameter

$b_{\mathrm{i}-\mathrm{i}}$ : phase-independent PR-EOS pure parameter

$H$ : Henry's law coefficient

$K_{\mathrm{i}-\mathrm{j}}$ : PR-EOS binary interaction parameter

$n c$ : number of components

$n p$ : number of experimental points

$p$ : Pressure

$R_{\mathrm{i}-\mathrm{j}}:$ ratio of mole fractions

$r_{\mathrm{i}-\mathrm{j}}:$ ratio of Raman signals

$S$ : fugacity criterion

$T$ : temperature

$u$ : standard uncertainty

$v$ : molar volume

$x$ : mole fraction of liquid or vapor phase

$\alpha_{\mathrm{i}}$ : PR-EOS parameter

$\varphi$ : fugacity coefficient

$\omega$ : PR-EOS acentric-factor

$\varepsilon$ : termination criterion

$\zeta$ : fugacity ratio

Superscript:

v: vapor phase; L: liquid phase; <,\#, +, lit: literature references

Subscript:

crit: critical; Eth: Ethanol; gas: pseudo-pure component gas; exp: experimental; calc: calculated; ter: ternary; Tx: isobaric; px: isothermal

\section{IX - References}

(1) Schnabel, T.; Vrabec, J.; Hasse, H. Henry's law constants of methane, nitrogen, oxygen and carbon dioxide in ethanol from 273 to $498 \mathrm{~K}$ : Prediction from molecular simulation. Fluid Phase Equilib. 2005, $233,134-143$.

(2) Malviya, A.; Vrabec, J. Henry's Law Constant of Nitrogen, Oxygen, and Argon in Ternary Aqueous Alcoholic Solvent Mixtures. J. Chem. Eng. Data [Online early access]. DOI: 10.1021/acs.jced.9b00571.

(3) Klima, T. C.; Braeuer, A. S. Vapor-liquid-equilibria of fuel-nitrogen systems at engine-like conditions measured with Raman spectroscopy in micro capillaries. Fuel 2019, 238, 312-319. 